\title{
Matrix Product Density Operators: Renormalization Fixed Points and Boundary Theories
}

\author{
J. I. Cirac ${ }^{1}$, D. Pérez-García ${ }^{2,3}$, N. Schuch ${ }^{1}$ and F. Verstraete ${ }^{4,5}$ \\ 1 Max-Planck-Institut für Quantenoptik, Hans-Kopfermann-Str. 1, D-85748 Garching, \\ Germany \\ ${ }^{2}$ Departamento de Análisis Matemático, Universidad Complutense de Madrid, Plaza de \\ Ciencias 3, 28040 Madrid, Spain \\ ${ }^{3}$ ICMAT, Nicolas Cabrera, Campus de Cantoblanco, 28049 Madrid, Spain \\ ${ }^{4}$ Department of Physics and Astronomy, Ghent University \\ 5 Vienna Center for Quantum Technology, University of Vienna
}

\begin{abstract}
We consider the tensors generating matrix product states and density operators in a spin chain. For pure states, we revise the renormalization procedure introduced in [F. Verstraete et al., Phys. Rev. Lett. 94, 140601 (2005)] and characterize the tensors corresponding to the fixed points. We relate them to the states possessing zero correlation length, saturation of the area law, as well as to those which generate ground states of local and commuting Hamiltonians. For mixed states, we introduce the concept of renormalization fixed points and characterize the corresponding tensors. We also relate them to concepts like finite correlation length, saturation of the area law, as well as to those which generate Gibbs states of local and commuting Hamiltonians. One of the main result of this work is that the resulting fixed points can be associated to the boundary theories of two-dimensional topological states, through the bulkboundary correspondence introduced in [J. I. Cirac et al., Phys. Rev. B 83, 245134 (2011)].
\end{abstract}

\section{Introduction}

Tensor networks offer concise descriptions of certain many-body quantum states in lattices. They thus consitute the basis of several numerical algorithms to solve strongly correlated systems problems $[1,2]$, like the density matrix renormalization group technique $[3,4]$. They have also proven to be very useful in the description of a variety of phenomena, including symmetry [5] or gauge invariance [6,7], and topologogical [8,9], and symmetry protected order [10].

Matrix product states (MPS), the tensor network states naturally describing one dimensional systems, are by now relatively well understood and characterized. In particular, they have given rise to the classification of gapped phases

Preprint submitted to Elsevier

November 16, 2016

(C) 2016. This manuscript version is made available under the Elsevier user license http://www.elsevier.com/open-access/userlicense/1.0/ 
in one-dimensional systems $[11,12,13]$. A key technique that has allowed to achieve this goal is the renormalization procedure introduced in [14] (see also [11]). The corresponding fixed points turn out to have a very simple structure, which indeed leads to the mentioned classification. In some sense they describe the coarse-grained behavior of all possible MPS, but with the peculiarity of having zero correlation length, as it should be expected for any renormalization procedure applied to gapped phases. Renormalization procedures are also at the realm of the multi-scale entanglement renormalization states [15], describing critical phenomena.

The extension of tensor network renormalization methods to higher dimensions is not a simple task. Simple renormalization procedures have been introduced in [16, 17], and the corresponding fixed point sets have been analyzed to some extent [18]. In particular, toric code [19] and string net states [20] belong to those sets. They have zero correlation length and possess topological order, which has been extensively investigated using projected entangled-pair states (PEPS) [21, 22], the natural generalization of MPS to higher spatial dimensions.

In this work we first thoroughly revisit the fixed points of the renormalization procedure introduced in [14] for one-dimensional spins and then extend the corresponding formalism to mixed states; that is, first we concentrate on MPS and then on matrix product density operators (MPDO). In this context, we define concepts like zero correlation length, saturation of the area law, as well as local commuting Hamiltonians, and connect them to the characterization of the fixed points. The main result of this work is the characterization of what we call renormalization fixed points (RFP) for MPDO. As we will see, they give rise to an algebraic structure that directly connects with fusion categories and string net states [20,23]. This is, indeed, what one could expect given the existing bulkboundary correspondence in PEPS, if we describe the boundary theory in terms of a MPDO [24]. Thus, some of the RFP can be interpreted as the boundaries of string net states. In this sense, the MPDO we find can be understood [26] as the product of a Gibbs state of a local commuting Hamiltonian with a nonlocal projector (which commutes with the Gibbs state), and which reflects an anomaly [25]. This can be interpreted as the consequence of the topological order of a two-dimensional theory at the boundary.

A basic tool of the characterization of the RFP, both for pure and mixed states, is the so-called canonical form of MPS [27]. In this paper we also carefully revise this notion, and derive some other results that are required for the formal proofs of our statements. In fact, since we introduce many definitions and such statements, and the corresponding proofs are sometimes involved, we have structured the paper as follows. First we introduce the main definitions and theorems, and we leave the technical material for the appendices. The paper starts out with the definition and characterization of canonical forms that include both MPS and MPDO, and in the next two sections we concentrate on the RFP of MPS and MPDO, respectively. 


\section{Matrix Product Vectors}

\subsection{Definition}

The main object of study here is a tensor, $A$, with coefficients $A_{\alpha, \beta}^{i}$, where $i=1, \ldots, d$ and $\alpha, \beta=1, \ldots, D$. We denote by $H_{d}$ a Hilbert space of finite dimension $d$, and by $\{|i\rangle\}_{i=1}^{d}$ an orthonormal basis in $H_{d}$. The tensor $A$ defines a family of vectors:

Definition 2.1. The vectors

$$
\left|V^{(N)}(A)\right\rangle=\sum_{i_{1}, \ldots, i_{N}=1}^{d} \operatorname{tr}\left(A^{i_{1}} \ldots A^{i_{N}}\right)\left|i_{1}\right\rangle \otimes \ldots \otimes\left|i_{N}\right\rangle \in H_{d}^{\otimes N}
$$

for $N=1,2,3, \ldots$ are the matrix product vectors generated by $A$. We call $D, d<\infty$ the bond dimension and the physical dimension, respectively.

We will omit the tensor products, and thus write $\left|i_{1}, i_{2}\right\rangle$ instead of $\left|i_{1}\right\rangle \otimes$ $\left|i_{2}\right\rangle$. Sometimes, we will use round kets and Greek letters, eg. $\mid \alpha$ ), to denote the elements of an orthonormal basis of vectors acting on the indices that are contracted in order to generate the MPV.

For the moment, we do not specify whether each MPV represents a state, in which case we will have a matrix product state (MPS), or an operator, in which case we will have a matrix product operator (MPO). We will denote by $\mathcal{V}(A)$ the family of MPV generated by $A$, i.e.

$$
\mathcal{V}(A)=\left\{\left|V^{(N)}(A)\right\rangle\right\}_{N \in \mathbb{N}} .
$$

The map

$$
A \rightarrow \mathcal{V}(A)
$$

is not one-to-one. In fact, different tensors may generate the same family of vectors, i.e. $\left|V^{(N)}(A)\right\rangle=\left|V^{(N)}(B)\right\rangle$. In the following sections we will analyze the relations among the tensors $A$ generating the same MPV's. In particular, we will show that one can always express a tensor in a canonical form so that one can easily compare the MPV's generated by different tensors in that form. This will be a key element of our analysis in the following sections.

By definition, the vector $V^{(N)}(A)$ is translationally invariant. One can also consider different matrices corresponding to different subsystems. However, here we will always deal with MPV of this form, and thus drop the word translationally invariant. Apart from that, in order not to overload the notation, we will drop the index $N$ and/or the dependence on $A$ in $V^{(N)}(A)$ and denote it simply by $V$ whenever this causes no confusion (or the corresponding family by $\mathcal{V}$ ). We will denote by $A^{i}$ the $D \times D$ matrices whose matrix elements are

precisely the coefficients $A_{\alpha, \beta}^{i}$ of the tensor $A$, and by $\mathcal{M}_{D \times D}$ the whole set of $D \times D$ matrices. 


\subsection{Graphical representations}

Since we will deal with various tensors contracted in different forms, the notation may become cumbersome. Thus, it will be useful to use a graphical representation in certain occasions. We will write a tensor in terms of a box with lines representing the different indices. For instance, the tensor $A$ generating MPV will be represented by

$$
A=-\frac{1}{A}
$$

where the line pointing up represents the physical index, and the other two the auxiliary indices. The contraction of two tensors will be represented by joining the lines of the corresponding indices. For instance, the coefficients of the MPV (1) in the basis $\left|i_{1}, \ldots, i_{N}\right\rangle$ will be represented as

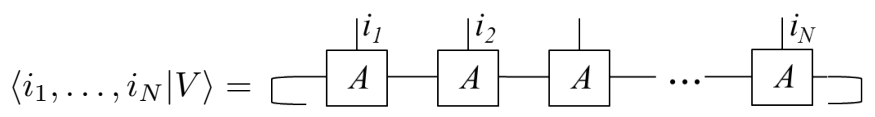

The meaning of this expression is that each coefficient can be written by multiplying the matrix $A^{i_{N}}$ by $A^{i_{N-1}}$, then by $A^{i_{N-2}}$, and so on. The curly lines at the end indicate that we have to take the trace at the end (i.e., contract the first with the last index after the matrix multiplication). We will abuse notation and simply write

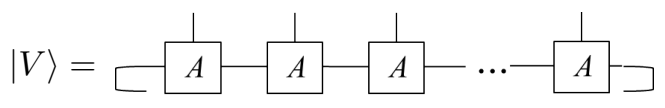

without explicitly writing the indices $i_{n}$.

Furthermore, since we will have to represent relative complex expressions with tensors, some of the lines will have to cross. If we write a full circle in the crossing, this will mean a delta function. For instance

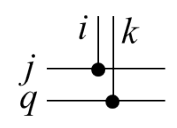

means that the indices on the right and the left coincide, and that $i=j$ and $k=q$ (but not that $i=k)$.

\subsection{Canonical forms}

Two tensors, $B$ and $C$, generate the same MPV if, for instance, all the matrices $B^{i}$ and $C^{i}$ are related by a similarity transformation

$$
B^{i}=X C^{i} X^{-1}
$$


where $X$ is a nonsingular matrix. Graphically,

$$
-\frac{1}{B}-=-x+X^{-1}
$$

We say that the tensors $B$ and $C$ are related by the gauge matrix $X$, and that the MPV are invariant under the similarity (gauge) transformation induced by $X$. But this is not the only way two tensors can generate the same MPV. It can also happen that all $B^{i}$ have a block upper triangular form, eg.

$$
B^{i}=\left(\begin{array}{cc}
B_{1}^{i} & B_{o}^{i} \\
0 & B_{2}^{i}
\end{array}\right)
$$

where $B_{k}^{i}$ are $D_{k} \times D_{k}$ matrices, and $B_{o}^{i}$ is a $D_{1} \times D_{2}$ matrix. The MPV generated by $B$ does not depend on $B_{o}^{i}$, so that for any such matrix we will have the same vector. In fact, there exists a subspace, $S_{1}$, of dimension $D_{1}$ which is left invariant under the action of all $B^{i}$, i.e. $B^{i} S_{1} \in S_{1}$. In order to lift this redundancy, it is convenient to select out of all the matrices $B^{i}$ generating the same MPV, those which have a simplified form. For instance, we can choose $B_{o}^{i}=0$, and use the invariance under the gauge transformation induced by a gauge matrix to specify certain properties of the $B^{i}$. In the following we will systematize this procedure to define canonical forms of MPV's.

First of all, let us assume that there exist one or more subspaces that are invariant under the action of all $B^{i}$. Let us denote by $S_{1}$ one such subspace, of dimension $D_{1}$, which does not contain any other invariant subspace, and let us denote by $P_{1}\left(Q_{1}=\mathbb{1}-P_{1}\right)$ the orthogonal projector onto $S_{1}\left(S_{1}^{\perp}\right)$. We have

$$
B^{i} P_{1}=P_{1} B^{i} P_{1}, \quad Q_{1} B^{i}=Q_{1} B^{i} Q_{1} .
$$

We consider now $Q_{1} B^{i} Q_{1}$ and proceed in the same form: identify an invariant subspace $S_{2} \subseteq S_{1}^{\perp}$ that does not contain any invariant subspace, denote by $P_{2}$ the corresponding projector, by $D_{2}$ the dimension of $S_{2}$, and $Q_{2}=\mathbb{1}-P_{1}-P_{2}$. In general, denoting by

$$
Q_{l}=\mathbb{1}-\sum_{k=1}^{l} P_{k},
$$

we have that

$$
Q_{l} B^{i} P_{l+1}=P_{l+1} B^{i} P_{l+1}, \quad Q_{l} B^{i}=Q_{l} B^{i} Q_{l} .
$$

After a finite number of steps, $r$, there will be no (non-trivial) invariant subspace anymore. The matrices $\left\{A^{i}\right\}$, defined as follows

$$
A^{i}=\sum_{k=1}^{r} P_{k} B^{i} P_{k}=\oplus_{k=1}^{r} \mu_{k} A_{k}^{i},
$$

generate the same family of MPV, $\mathcal{V}$. Here, $r$ is the number of blocks, the $\mu_{k}$ are complex numbers, and $A_{k}^{i}$ are $D_{k} \times D_{k}$ matrices with $\sum_{k} D_{k} \leq D$ (i.e., 
there can be zero blocks). We associate each tensor $A_{k}$ a completely positive map $(\mathrm{CPM}), \mathcal{E}_{k}$, defined through

$$
\mathcal{E}_{k}(X)=\sum_{i=1}^{d} A_{k}^{i} X A_{k}^{i \dagger} .
$$

The values of $\mu_{k}$ are chosen such all these maps have spectral radius equal to 1 .

As it was shown in [27], each CPM $\mathcal{E}_{k}$ has a unique eigenvalue $\lambda=1$. However, there may be other eigenvalues of magnitude one, $e^{i 2 \pi q / p}$, with $p, q$ integers, $\operatorname{gcd}(q, p)=1$, and where $p$ is a divisor of $D$. They correspond to so-called $p$-periodic vectors [28]. In this context, it is very convenient to define the procedure of blocking, which will also play a role when we talk about renormalization procedures. By blocking tensors $B$ we mean defining a tensor $C$ such that the corresponding matrices are obtained by the $p$-fold products of the original ones, i.e. $C^{i}=B^{i_{1}} \ldots B^{i_{p}}$, where the index $i$ is contains all the $i_{k}$, $k=1, \ldots, p$. Graphically,

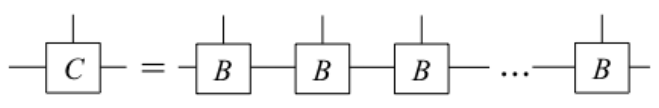

If there are $t p$-periodic vectors, with $p=p_{1}, \ldots, p_{t}$, by blocking $\operatorname{lcm}\left(p_{1}, \ldots, p_{t}\right)$ systems, one obtains a vector without $p$-periodic ones. Since in the present work we will be mostly interested in MPVs obtained after a renormalization procedure which will block large numbers of spins anyway, we will be able to assume that for each $\tilde{\mathcal{E}}_{k}$ there is a unique eigenvalue of magnitude (and value) equal to one. Note that, when discussing MPOs, we may not be allowed to block tensors in some special ways, so that in that case we will have to ensure that there are no $p$-periodic vectors in case we want to use the canonical forms defined below.

Definition 2.2. We call a tensor, $A$, a normal tensor (NT) if: (i) there exist no non-trivial projector $P$ such that $A^{i} P=P A^{i} P$; (ii) its associated $C P M$, has a unique eigenvalue of magnitude (and value) equal to its spectral radius, which is equal to one. We call $|V\rangle$ normal matrix product vector (NMPV) if it is generated by a normal tensor.

Definition 2.3. We say that a tensor, $A$, is in a canonical form $(C F)$, if

$$
A^{i}=\oplus_{k=1}^{r} \mu_{k} A_{k}^{i}
$$

and the tensors $A_{k}$ are NT.

Note that we are not considering here normalized states, so that we can always choose $\left|\mu_{k}\right| \leq 1$ and at least one of them equals one, something which we will assume from now on. The next propositions follow from the above procedure. 
Proposition 2.4. After blocking, for any tensor, B, it is always possible to obtain another one, $A$, in $C F$ and generating the same $\mathcal{V}$.

Proposition 2.5. A tensor, $A$, is in $C F$ if it has no p-periodic vectors and for every projector, $P$, fulfilling $P A^{i}=P A^{i} P$, we also have $A^{i} P=P A^{i} P$.

The CF of a tensor (17) immediately implies that

$$
\left|V^{(N)}(A)\right\rangle=\sum_{k=1}^{r} \mu_{k}^{N}\left|V^{(N)}\left(A_{k}\right)\right\rangle,
$$

Note that two or more families $\mathcal{V}\left(A_{j}\right)$ may be the same, or related by a phase. This happens, for instance, if two of the tensors, say $A_{j}$ and $\tilde{A}_{k}$, satisfy

$$
\tilde{A}_{k}=e^{i \phi_{k}} X_{k} A_{j} X_{k}^{-1}
$$

for some invertible $X_{k}$ and phase $\phi_{k}$. This case has to be treated with some care, and for that we will introduce the following

Definition 2.6. The tensors $A_{j}(j=1, \ldots, g)$ form a basis of normal tensors (BNT) of a tensor $A$ if: (i) the $A_{j}$ are NT; (ii) for each $N, V^{(N)}(A)$ can be written as a linear combination of $V^{(N)}\left(A_{j}\right)$; (iii) there exists some $N_{0}$ such that for all $N>N_{0}, V^{(N)}\left(A_{j}\right)$ are linearly independent.

Note that for any tensor, there always exists a BNT. In Appendix A we show how to do that in the proof of the following characterization of BNT.

Proposition 2.7. The tensors $A_{j}(j=1, \ldots, g)$ form a BNT of $A$ iff: (i) for all NT appearing in its CF (17), $\tilde{A}_{k}$, there exists a $j$, a non-singular matrix, $X_{k}$, and some phase, $\phi_{k}$, such that (19) holds; (ii) the set is minimal, in the sense that for any element $A_{j}$, there is no other $j^{\prime}$ for which (19) is possible.

We can write now the matrices of any tensor, $A$, in $\mathrm{CF}$ in terms of a BNT, $A_{j}$, as

$$
\begin{aligned}
A^{i} & =X\left[\oplus_{j=1}^{g}\left(M_{j} \otimes A_{j}^{i}\right)\right] X^{-1} \\
& =\oplus_{j=1}^{g} \oplus_{q=1}^{r_{j}} \mu_{j, q} X_{j, q} A_{j}^{i} X_{j, q}^{-1},
\end{aligned}
$$

where $M_{j}$ is a diagonal matrix with coefficients $\mu_{j, q}$, and

$$
X=\oplus_{j=1}^{g} \oplus_{q=1}^{r_{j}} X_{j, q}
$$

so that

$$
\left|V^{N}(A)\right\rangle=\sum_{j=1}^{g}\left(\sum_{q=1}^{r_{j}} \mu_{j, q}^{N}\right)\left|V^{(N)}\left(A_{j}\right)\right\rangle .
$$

We will express (20a) graphically as follows

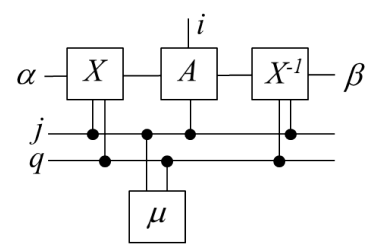


where

$$
A_{j}^{i}=-\underbrace{i}_{\frac{j}{A}}
$$

are the BNT. We have included in (23) all the indices, in order to make the relation with (20a) more evident. Note that we have represented the indices $j$ and $q$ by lines, and thus replaced the direct sums by tensor products. In reality, the values over which the indices $q, \alpha$, and $\beta$ run will generically depend on $j$; for instance, $q=1, \ldots, r_{j}$. Thus, the graphical representation should not be taken literally.

Now we introduce a basic definition in the context of MPS, on which many of the special properties of those states rely.

Definition 2.8. ANT A is called injective, if the matrices $A^{i}$ span the whole set of $\mathcal{M}_{D \times D}$ matrices. We say that a tensor $A$ is in block injective canonical form (biCF) if it is in $C F$, and for each element $X \in \oplus_{j=1}^{g} \mathcal{M}_{D_{j} \times D_{j}}$ there exists a vector, $c(X)$, such that $X=\sum_{i} c_{i}(X) \tilde{A}^{i}$, where $\tilde{A}^{i}:=\oplus_{i=1}^{g} A_{j}^{i}$, and $A_{j}$ are $a$ $B N T$ of $A$.

The first definition is equivalent to the existence of another tensor, $A^{-1}$, such that $\sum_{i=1}^{d}\left(A^{i}\right)_{\alpha, \beta}\left(A^{-1, i}\right)_{\alpha^{\prime}, \beta^{\prime}}=\delta_{\alpha, \alpha^{\prime}} \delta_{\beta, \beta^{\prime}}$, and the second one to the existence of tensors $A_{j}^{-1}$ such that $\sum_{i=1}^{d}\left(A_{j}^{i}\right)_{\alpha, \beta}\left(A_{j^{\prime}}^{-1, i}\right)_{\alpha^{\prime}, \beta^{\prime}}=\delta_{\alpha, \alpha^{\prime}} \delta_{\beta, \beta^{\prime}} \delta_{j, j^{\prime}}$; graphically,

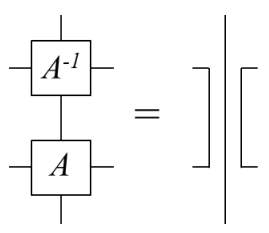

According to the quantum version of Wieland's theorem [29], after blocking at most $D^{4}$ times, every NT becomes injective. We will use this property several times in the coming sections. The definition of biCF basically says that acting on the physical index we have access to each and every element of every BNT.

Furthermore, in [27] it was shown that after $3(L+1)(D-1)$ blockings, any tensor $A$ becomes block injective, where $L$ is the number of blockings after which each NT becomes injective. Thus we have

Proposition 2.9. After blocking at most $3 D^{5}$ spins, any tensor $A$ in $C F$ is in biCF.

We are then ready to state the main result of this section, that we call The Fundamental Theorem of Matrix Product Vectors. It clarifies which is the freedom for two tensors $A$ and $B$ in CF, to generate families of MPV, $\mathcal{V}_{a, b}$, such that for each $N$ they are proportional or equal to each other 
Theorem 2.10. Let $A$ and $B$ be two tensors in $C F$, with BNT $A_{k_{a}}^{i}$ and $B_{k_{b}}^{i}$ $\left(k_{a, b}=1, \ldots, g_{a, b}\right)$, respectively. If for all $N, A$ and $B$ generate $M P V$ that are proportional to each other, then: (i) $g_{a}=g_{b}=: g$; (ii) for all $k$ there exists a $j_{k}$, phases $\phi_{k}$, and non-singular matrices, $X_{k}$ such that $B_{k}^{i}=e^{i \phi_{k}} X_{k} A_{j_{k}}^{i} X_{k}^{-1}$.

Corollary 2.11. If two tensors $A$ and $B$ in $C F$ generate the same $M P V$ for all $N$ then: (i) the dimensions of the matrices $A^{i}$ and $B^{i}$ coincide; (ii) there exists an invertible matrix, $X$, such that

$$
A^{i}=X B^{i} X^{-1} .
$$

The justification of the name came from the wide and important uses of that result. Besides being the key technical tool in this paper, it has been also the key result in the characterization of symmetries and string order parameters of MPS [5], the classification of phases in 1D systems [11, 12], or the construction of string nets with PEPS [23].

\section{Pure States: Renormalization of Matrix Product States}

Here, we consider $N$ spins in a 1D chain, and a translationally invariant MPS. That is, now $H_{d}$ is the Hilbert space for each spin, and thus the vectors $V^{(N)}(A)$ represent states of those spins. We are interested in defining a renormalization procedure whose fixed points will be the central subject of this section. The renormalization flow will encompass the idea of coarse-graining transformations in space, as it appears in some versions of the standard renormalization group methods. However, it is defined in the space of tensors generating the MPS, as it should affect the whole family of states generated by the tensors. We will analyze the fixed points of that flow, ie. the renormalization fixed points (RFP), as well as their connection with other concepts that naturally appear in the context of many-body systems, like zero correlation length (ZCL), or ground states of frustration-free, commuting Hamiltonians (GSCH). We will finally show how all those notions are equivalent in the main theorem of this section. The other main result of this section characterizes being an RFP in the structure of the local tensor $A$.

\subsection{Renormalization Flow and Renormalization Fixed Point}

We consider the renormalization procedure introduced in [14]. There the idea was to separate MPS in classes such that their elements, when blocking spins, were related by local unitary transformations. The blocking of spins in this procedure gives rise to a coarse grained picture of MPS, and defines a flow in the set of tensors generating the MPS. In the limit $m \rightarrow \infty$, this flow converges

to a special set of tensors, whose elements can be considered as the fixed points of the renormalization procedure.

In renormalization theory one is interested in global features of a system and hence, in order to define the renormalization flow, we will identify those states 
that are related by local unitary transformations. That is, tensors $A$ and $\tilde{A}$ are considered equivalent if they are related by an isometry $U$ :

$$
\begin{gathered}
\frac{1}{U} \\
-\tilde{A} \\
-1 \\
-
\end{gathered}
$$

This divides the set of tensors in equivalence classes. We will define now the basic step of the renormalization procedure that maps a class of tensors into another class. We will do that by considering how one element of the class is mapped into another element of the new class, as this definition respects the equivalence classes. In particular, let us consider a tensor $B$ and perform the blocking of two spins as defined in the previous section. Thus, we have the new tensor, $C$, with components $C_{\alpha_{1}, \alpha_{2}}^{i_{1}, i_{2}}=\left(\alpha_{1}\left|B^{i_{1}} B^{i_{2}}\right| \alpha_{2}\right)$. This can be viewed as a matrix, $C_{\alpha}^{i}$, where $i=\left(i_{1}, i_{2}\right)$ and $\alpha=\left(\alpha_{1}, \alpha_{2}\right)$ are combined indices. Since the rank of this matrix, $d_{B} \leq D^{2}$ (the possible values taken by $\alpha$ ), we can always find a $d^{2} \times d_{B}$ isometry, $U$, with $U^{\dagger} U=\mathbb{1}_{d_{B}}$ and write $C=U A$. That is,

$$
B^{i_{1}} B^{i_{2}}=\sum_{j=1}^{d_{B}} U_{i, j} A^{j}
$$

for some matrices $A^{j}$ and $d_{B} \leq D^{2}$. The flow just maps the class of tensors represented by $B$ in that represented by $A$.

We are interested in the form of the tensor $A$ with matrices $A^{j}$ that appear as limits of such renormalization flow when $m \rightarrow \infty$. By the very definition (28) of the renormalization flow, one clearly expects the following

Theorem 3.1. A tensor $A$ appears as a limit in the above renormalization flow if and only if

$$
A^{i_{1}} A^{i_{2}}=\sum_{i_{1}, i_{2}, j} U_{\left(i_{1}, i_{2}\right), j} A^{j_{1}}
$$

for an isometry $U$.

Graphically,

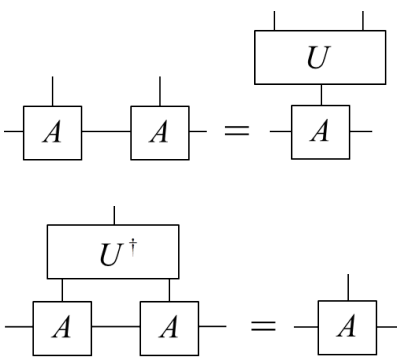


and, schematically

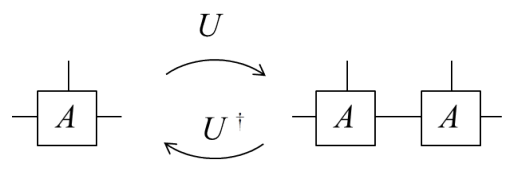

Moreover, it will be also shown in Appendix B that if we start with a tensor $B$ in $\mathrm{CF}$, the above renormalization flow always converges. This result motivates the following

Definition 3.2. A tensor $A$ is called a renormalization fixed point (RFP) if it verifies (29), that is, if one can obtain two tensors out of one (and vice versa) by the action of an isometry in the physical index.

\subsection{Zero correlation length}

The renormalization flow defined above does not change the state, but just redefines the spins as it makes them larger and larger by blocking them. Thus, one should expect that if there is any length scale in the system, it will disappear at the end of the flow. In this section we will show how this is captured at the RFP.

\subsubsection{Correlations independent of the distance and local orthogonality}

Perhaps the main notion of distance that naturally appears in the context of the many-body states we are dealing with is the correlation length. This is roughly defined as the typical length at which correlations decay. According to what we expressed above, the RFP should have zero correlation length. This statement can be made more specific as follows. Let us denote by $R_{n_{1}, n_{2}}$ the set of spins with $n_{1} \leq n \leq n_{2}$. Then

Definition 3.3. We say that $\Psi$ has correlations independent of the distance (CID) if for any pair of observables, $O_{n_{1}, n_{1}^{\prime}}, O_{n_{2}, n_{2}^{\prime}}$, acting on any disjoint regions $R_{n_{1}, n_{1}^{\prime}}$ and $R_{n_{2}, n_{2}^{\prime}}$, respectively,

$$
\left\langle\Psi\left|O_{n_{1}, n_{1}^{\prime}} O_{n_{2}, n_{2}^{\prime}}\right| \Psi\right\rangle=\left\langle\Psi\left|O_{n_{1}, n_{1}^{\prime}} O_{n_{2}+\Delta, n_{2}^{\prime}+\Delta}\right| \Psi\right\rangle
$$

for all $n_{1}^{\prime}-n_{2}+1<\Delta<N-n_{2}^{\prime}$.

Even though this definition is probably the most natural one, there are states with CID which keep other type of lengths (and hence cannot be RFP) as the following trivial example shows.

Example 3.4. Let us consider the state

$$
|\Psi\rangle=|0, \ldots, 0\rangle+|+, \ldots,+\rangle,
$$

where $|+\rangle=(|0\rangle+|1\rangle) / \sqrt{2}$. The corresponding matrices are

$$
A^{0}=\left(\begin{array}{cc}
1 & 0 \\
0 & 1 / \sqrt{2}
\end{array}\right), \quad A^{1}=\left(\begin{array}{cc}
0 & 0 \\
0 & 1 / \sqrt{2}
\end{array}\right) .
$$


This state is permutationally invariant, and thus it trivially satisfies (33). However, it does not fulfill (29), and thus it is not a RFP. In fact, if we block $n$ spins we will have

$$
|\Psi\rangle=|\tilde{0}, \ldots, \tilde{0}\rangle+|\tilde{+}, \ldots, \tilde{+}\rangle,
$$

where $|\tilde{0}\rangle=|0, \ldots, 0\rangle$ and $|\tilde{+}\rangle=|+, \ldots,+\rangle$. Although this state looks very similar to the original one, now the scalar product $\langle\tilde{0} \mid \tilde{+}\rangle$ decreases exponentially with $n$. Thus, if we think in terms of renormalization, the state (34) cannot be a renormalization fixed point despite the fact that has CID.

The previous example illustrates that if we have CID and we keep on blocking, the states generated by the different elements of the BNT will look locally orthogonal. This property can be defined as follows

Definition 3.5. We say that two tensors, $A_{j}, A_{j^{\prime}}$ are orthogonal if

$$
\sum_{i} A_{j}^{i} \otimes \bar{A}_{j^{\prime}}^{i}=0
$$

We will say that a MPV is locally orthogonal (LO) if the associated BNT are orthogonal.

Definition 3.6. We will say that a tensor $A$ has $Z C L$ if the generated family of MPS is LO and has CID.

The property of ZCL for a tensor can be easily characterized using the socalled transfer matrix.

Definition 3.7. Given a tensor A, generating a family of $M P V$, we define the transfer matrix

$$
\mathbb{E}=\sum_{i} A^{i} \otimes \bar{A}^{i}=\frac{\sqrt{\bar{A}}-}{-A}
$$

The transfer matrix is the standard tool to calculate expectation values. For any pair of observables, $O_{1,2}$, acting on subsystems $s_{n_{1,2}}$, respectively,

$$
\left\langle V\left|O_{1} O_{2}\right| V\right\rangle=\operatorname{tr}\left(\mathbb{E}_{O_{1}} \mathbb{E}^{n_{2}-n_{1}-1} \mathbb{E}_{O_{2}} \mathbb{E}^{N+n_{1}-n_{2}-1}\right),
$$

where $\mathbb{E}_{O}$ is a matrix that depends on the matrices $A^{i}$ as well as on the operator $O$.

Note that if $\mathbb{E}^{2}=\mathbb{E}$, then one will have CID. In fact, this condition is equivalent to ZCL:

Theorem 3.8. A tensor $A$ in $C F$ has $Z C L$ if and only if $\mathbb{E}^{2}=\mathbb{E}$. 


\subsection{Commuting parent Hamiltonians}

One can always build local Hamiltonians for which a MPS is a ground state. Among them, the so-called parent Hamiltonians play an important role, as they ensure the ground space is very much related to the structure of the tensors generating the MPS. They are additionally translationally invariant and frustration free; that is, a sum of positive operators that act on a neighborhood of each spin, and that annihilate the state. In this section we will show that if, additionally, those local operators commute with each other, then there is an intimate relation between their ground states and the RFP. In Appendix D we also derive a general relation between ground states of commuting parent Hamiltonians and the absence of correlations.

Let us consider a tensor $A$ generating a family of MPS. Let us take a block consisting of $L$ spins, and the subspace, $S_{L}$, spanned by

$$
\left|v_{\alpha, \beta}\right\rangle=\sum_{i_{1}, \ldots, i_{L}}\left(\alpha\left|A^{i_{1}} \ldots A^{i_{N}}\right| \beta\right)\left|i_{1}, \ldots, i_{N}\right\rangle
$$

Let us further assume that $d^{L}>D^{2} \geq \operatorname{dim}\left(S_{L}\right)$, so that $S_{L}^{\perp}$ is non-trivial. We denote by $P_{L}^{\perp}$ the projector onto that subspace and construct the Hamiltonian

$$
H_{L}^{(N)}=\sum_{j=0}^{N-1} \tau_{j}\left(P_{L}^{\perp}\right)
$$

where $\tau_{j}$ translates the spins by an amount $j$, so that it is translationally invariant (with periodic boundary conditions). Obviously, $\left|V^{(N)}\left(A_{j}\right)\right\rangle$ are ground states of $H_{L}^{(N)}$ with zero energy.

Definition 3.9. Given a tensor in $C F$, with $B N T A_{j}(j=1, \ldots, g)$, we say that (41) is a parent Hamiltonian if the ground state subspace is spanned by $\left|V^{(N)}\left(A_{j}\right)\right\rangle$ for all $N>L$. A commuting parent Hamiltonian fulfills $\left[\tau_{j}\left(P_{L}\right), P_{L}\right]=$ 0 for all $j=1, \ldots, L-1$. A nearest-neighbor parent Hamiltonian has $L=2$.

A direct consequence of Proposition 2.9 [27] is that for any $A$ one can always find a parent Hamiltonian with $L \leq 3 D^{5}$ independent of the tensor $A$ (just of its bond dimension). Thus, if we block spins it is always possible to find a nearestneighbor parent Hamiltonian. In the following section we will establish a close relation between RFP and the ground states of nearest-neighbor commuting parent Hamiltonians (NNCPH).

\subsection{Main theorems}

We can now state the main results of the section. In Theorem 3.10, we show the announced equivalence between being a RFP, ZCL, and NNCPH. Theorem 3.11 gives a characterization of the tensors $A$ with the RFP property.

Theorem 3.10. The following properties are equivalent for a tensor $A$ in $C F$ generating a family of MPS. 
(i) RFP.

(ii) $Z C L$.

(iii) For all $N>2,\left|V^{(N)}(A)\right\rangle$ is a ground state of a $N N C P H$.

Theorem 3.11. A tensor $A$ in $C F$ is a RFP iff it can be written as

$$
A^{i}=\oplus_{j=1}^{g} \oplus_{q=1}^{r_{j}} \mu_{j, q} X_{j, q} \Lambda_{j} U_{j}^{i} X_{j, q}^{-1}
$$

where $\left|\mu_{j, q}\right|=1$, the matrices $\Lambda_{j}$ are diagonal, positive, and $\operatorname{tr}\left(\Lambda_{j}\right)=1$, and $U$ is an isometry in the sense that

$$
\sum_{i=1}^{s}\left(U_{j}^{i}\right)_{\alpha, \beta}\left(\bar{U}_{j^{\prime}}^{i}\right)_{\alpha^{\prime}, \beta^{\prime}}=\delta_{j, j^{\prime}} \delta_{\alpha, \alpha^{\prime}} \delta_{\beta, \beta^{\prime}}
$$

We can represent this graphically as

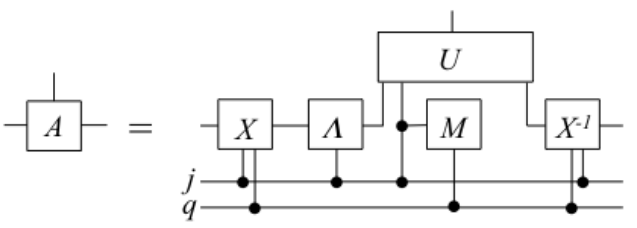

This expression has the following meaning. There are two indices, $j, q$, which give rise to the different blocks and are indicated in the graph. The index $j$ enumerates the elements of a BNT, whereas the $q$ gives different representations of the same NT. The tensors $X$, and $M$ depend on the values of those two indices, whereas $\Lambda$ only depends on $j$. The resulting matrices, $X_{j, q}$ and $\Lambda_{j}$ are multiplied and, together with $X_{j, q}^{-1}$, and the indices $j$ and $q$ give rise to the physical index, after applying the isometry $U$. Note that since the indices $j$ and $q$ connect the physical and virtual indices, up to the isometry, they give rise to a direct sum in both physical and virtual spaces.

The MPS generated by a RFP tensor $A$ in CF thus have the form

$$
\left|V^{(N)}(A)\right\rangle=\sum_{j=1}^{g}\left(\sum_{q=1}^{r_{j}} e^{i N \phi_{j, q}}\right)\left|V^{(N)}\left(A_{j}\right)\right\rangle,
$$

where its basic vectors give

$$
\left|V^{(N)}\left(A_{j}\right)\right\rangle=U^{\otimes N}\left|\varphi_{j}\right\rangle^{\otimes N}
$$

with

$$
\left|\varphi_{j}\right\rangle=\sum_{m_{j}=1}^{d_{j}} \lambda_{m_{j}}\left|m_{j}, m_{j}\right\rangle .
$$

Note that one can understand the state by taking two spins per node, $a_{n}$ and $b_{n}$ : the state $\varphi_{j}$ is shared by any two spins in neighboring nodes, $b_{n}$ and $a_{n+1}$, 
whereas the isometry $U$ acts on the two spins at each node, $a_{n}$ and $b_{n}$. The state can be graphically represented as

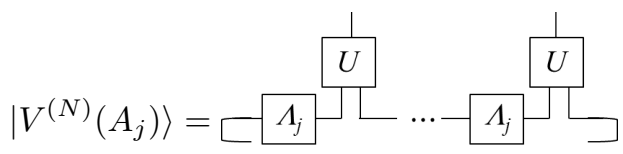

Corollary 3.12. The elements of a BNT corresponding to a tensor $A$ that is RFP have the form

$$
A_{j}=X_{j} \Lambda_{j} U_{j}^{i} X_{j}^{-1}
$$

where $U$ is an isometry fulfilling (43).

\subsection{Saturation of the area law}

We finish this section by noticing that there exists another property that is implied by RFP tensors, and which is connected to the entanglement content of the states. We consider the von Neumann entropy of a block of $L$ spins contained in $\left|V^{(N)}(A)\right\rangle$. This entropy is defined as

$$
S_{L}^{(N)}(A)=-\operatorname{tr}\left[\rho_{L} \log _{2}\left(\rho_{L}\right)\right]:=S\left(\rho_{L}\right)
$$

where $\rho_{L}$ is the corresponding reduced state of $L$ spins. Since all MPS fulfill the area law, it is upper bounded by a constant. Furthermore, using the strong subadditivity inequality, translationally invariance, and the fact that $S_{(N)}^{N}=0$, it is simple to show that $S_{L}^{(N)}$ is an increasing function of $L$ for $N>2 L$. Thus, in the limit $N \rightarrow \infty$ it will saturate to a constant. This motivates the following definition

Definition 3.13. We say that a tensor $A$ saturates the area law $(S A L)$ if the generated MPS fulfill $S_{1}^{(N)}(A)=S_{2}^{(N)}(A)=\ldots S_{N / 2}^{(N)}(A)$

We can establish a relationship between SAL and RFP as follows (the result is a trivial consequence of the general case of mixed states that we will analyze later on):

Proposition 3.14. If a tensor $A$ in $C F$ is RFP then it is $S A L$.

Note that, as it will be clear from the next section when we study mixed states, the converse is not necessarily true. However, in that case we will impose ZCL as well in order to assess the equivalence, at least for some class of tensors. In the present pure case it does not make sense to add this condition, since it is already equivalent to RFP.

\section{Mixed States}

As in the previous section we consider spins in a 1D chain, but now we focus on mixed states. That is, we consider a tensor, $M$, that generates translationally 
invariant density operators acting on the spins called matrix product density operators (MPDO) [30, 31]. The tensor $M$ has four indices, two auxiliary (as a MPV) and two physical (as an operator acting on the Hilbert space of the spins). That is, for the MPDO corresponding to $N$ spins we write

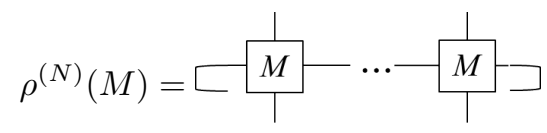

The arrows indicate that the density operator is acting from bottom to top. Those operators are hermitian and positive semidefinite, ie. $\rho^{(N)}(M)=\rho^{(N)}(M)^{\dagger} \geq$ 0 .

In the following, we will define the renormalization fixed points (RFP) of the tensors $M$ generating families of MPDO. We will also analyze their connection with those that have "no length scale", by studing states with ZCL and also that saturate the area law (SAL) for the mutual information. As we will see, those conditions are not equivalent, but together coincide with the definition of RPF for some particular cases. We will characterize the tensors corresponding to ZCL and SAL and, finally, the RPF for the general case.

\subsection{Renormalization flow and renormalization fixed points}

As opposed to the previous section, it is in principle not clear what should be the right definition of RFP in this context, apart from the fact that it should capture the hand-waving intuition of "blocking until there is no length scale left in the system". The problem we face can be explained as follows. If we define a renormalization procedure as we did before, the dimension of the Hilbert space corresponding to one site can grow indefinitely. In the pure state case, due to the area law the relevant part of this space (i.e., the subspace where the state is supported) remains finite (its dimension is bounded by $D^{2}$ ), which allows one to describe the fixed points of the procedure within the MPV formalism. In the mixed case, this is not longer true (it is only the space of operators acting on that space which remains finite, but their action can involve the whole Hilbert space). Thus, in general, one should deal with local physical spaces whose Hilbert spaces have infinite dimensions, which takes us out of the MPV description.

A natural way around this problem is to define a renormalization flow that keeps the physical dimensions, very much in the spirit of standard renormalization group. This could be done in terms of a trace-preserving completely positive map (tpCPM), $\mathcal{S}$, that transforms the state of two spins, $\rho_{12}$ into a state of one, $\sigma_{1}$, but keeping the Hilbert space of the final spin equal to the original one: $\mathcal{S}\left(\rho_{12}\right)=\sigma_{1}$. This automatically induces a map in the tensors $M$ 
generating the MPDO. Defining

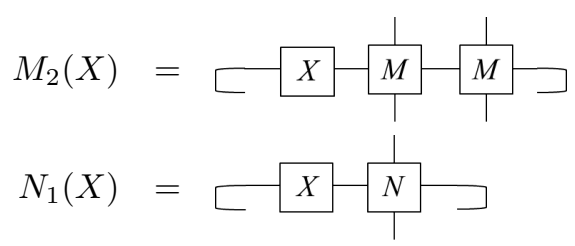

Then, we have $\mathcal{S}\left[M_{2}(X)\right]=N_{1}(X)$ for all $X$. The flow, as before, can be obtained by successive application of $\mathcal{S}$. The fixed point, a tensor $M$, should then satisfy

$$
\mathcal{S}\left[M_{2}(X)\right]=M_{1}(X) .
$$

However, notice that the map may truncate part of the information, and thus make the procedure irreversible. In order to restore the reversibility, we will require the existence of another tpCPM, $\mathcal{T}$ mapping the state of one spin into that of two, i.e. $\mathcal{T}\left[M_{1}(X)\right]=N_{2}(X)$, such that for the fixed points it also fulfills

$$
\mathcal{T}\left[M_{1}(X)\right]=M_{2}(X)
$$

for all $X$. This motivates the following

Definition 4.1. Given the tensor $M$, in $C F$, generating a family of $M P D O s$, we say that it is a RFP if there exist two tpCPM, $\mathcal{T}, \mathcal{S}$ acting on the physical indices, fulfilling (53) and (54).

Graphically,

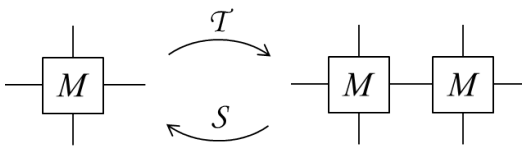

The first observation is that this definition coincides with Definition 3.2 in the particular case in which the MPDO is pure, i.e. an MPS. In that case, the tensor $M$ is given by

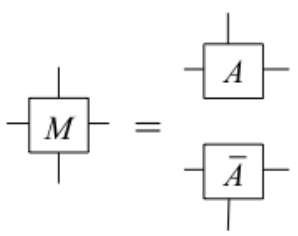

Definition 3.2 is equivalent to the existence of a unitary $U$ acting on two sites and a pure state $e$ on one site so that 


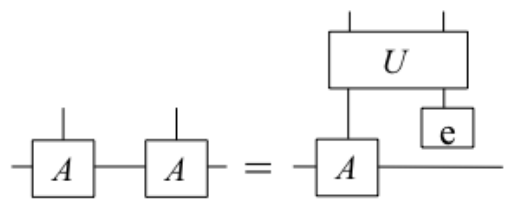

Then we obtain (55) for $M$ if we define $\mathcal{T}(X)=V(X \otimes|e\rangle\langle e|) V^{\dagger}$ and $\mathcal{S}(Y)=$ $\operatorname{tr}_{2}\left(V^{\dagger} Y V\right)$ where $\operatorname{tr}_{2}$ traces the second system. Graphically,
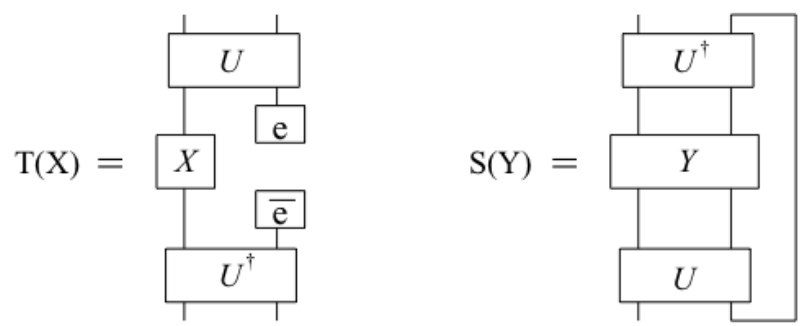

Definition 4.1 appears also very naturally in the context of boundary theories.

\subsection{Boundary theories}

Using the same tensor notation as introduced in Section 2.2, one can deal with systems arranged in 2D lattices (wlog the square lattice). If the tensors give rise to pure states, they are called Projected Entangled-Pair States (PEPS). That is, the tensor

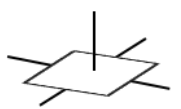

while contracted through a $2 \mathrm{D}$ square lattice of size $L_{H} \times L_{V}$ with periodic boundary conditions

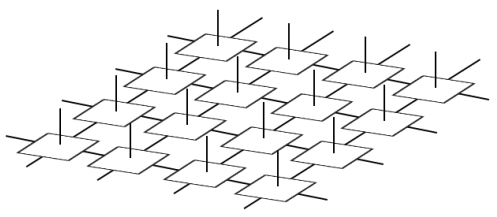

gives rise to a vector in $\left(\mathbb{C}^{d}\right)^{\otimes L_{H} L_{V}}$.

PEPS are known to be a good description of ground states of gapped local Hamiltonians and, since any ground state of a frutration-free commuting Hamiltonian is a PEPS, they are expected to represent exactly ground states of gapped RFP quantum phases in 2D. 
In [24], motivated by the seminal paper of Haldane and Li [32], the authors show an holographic correspondence for PEPS: an explicit isometry connecting the PEPS with a 1D mixed state living at the boundary. Interpreting such mixed state as $e^{-H_{b}}$, gives a 1D Hamiltonian $H_{b}$ which is called the boundary theory of the PEPS. Moreover, $e^{-H_{b}}$ is given explicitly in terms of the left and right fixed points of the transfer operator of the PEPS,

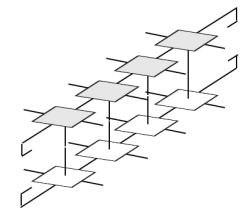

It is clear from the way one computes expectation values in PEPS that such fixed points encode the correlations present in the PEPS. In an abuse of notation, here we will call any such fixed point a boundary theory.

As in any holographic correspondence, the aim of studying boundary theories is to provide with a dictionary translating relevant properties of the bulk to physically meaningful properties of $H_{b}$. In $[24,26]$ numerical evidence has been provided that (i) a gapped bulk corresponds to $H_{b}$ having quasi-local interactions; and (ii) the existence of topological order is reflected in some anomaly present in $H_{b}$, in the sense that $e^{-H_{b}}$ is supported only in a subspace of the total Hilbert space that encodes the topological features of the bulk. The main results of this section can be seen as analytical proofs of such dictionary for the case of RFP.

From the point of view of boundary theories, there is a natural way to define RFP for mixed states, take a PEPS which is already the fixed point of some renormalization procedure and see how such property is transferred to the boundary. In this sense, let us consider a PEPS that is in an RFP, so that its tensor verifies the following RFP property:

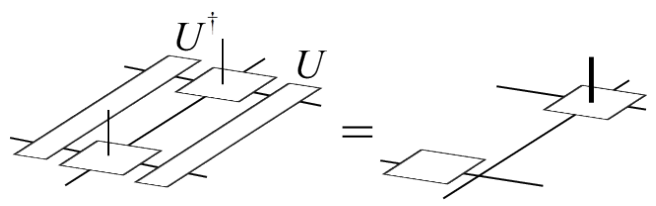

where equality means up to an isometry acting on the physical indices. In this case, the tensor associated to the boundary theory can be seen to be (using also that the PEPS is an RFP):

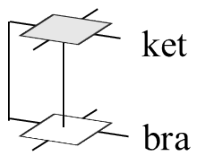


and property (62) maps to

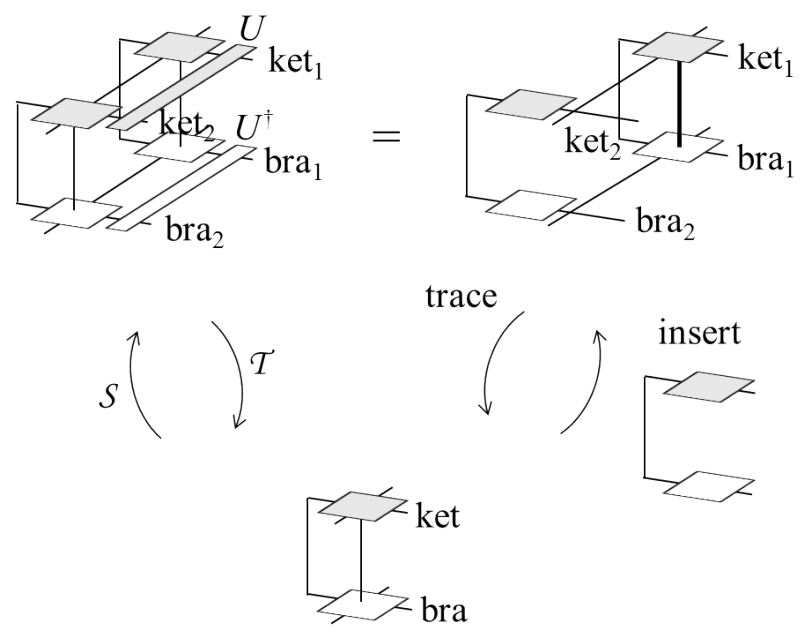

which gives the desired $T$ and $S$ of Definition 4.1.

\subsection{Zero correlation length}

We have seen that in the MPS case ZCL is equivalent to RFP. Since the pure case is a particular case of the mixed one, one may try to see whether the corresponding generalization of ZCL is enough to capture the RFP property.

Definition 4.2. A tensor $M$ generating $M P D O$ is said to have zero correlation length $(Z C L)$ if

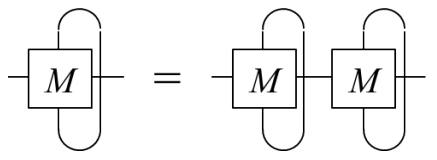

This is the natural extension of the pure state case of Theorem 3.8. Indeed, it is clear that if we compute correlation functions using a MPDO generated by a tensor $M$ with ZCL, they will be length-independent.

In order to characterize the tensors fulfilling this property, we will write the tensor $M$ generating MPDOs in terms of another tensor, $A$, generating a purification. ${ }^{1}$ Graphically, we have

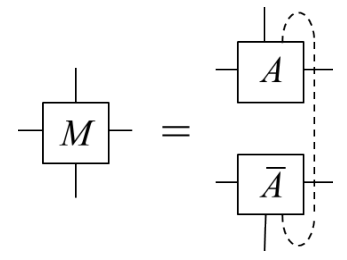

\footnotetext{
${ }^{1}$ This may not be always possible, see [33].
} 
In terms of the MPDO, the interpretation is that apart from the $N$ spins, we have $N$ ancillary systems, each of them associated to one spin, such that

$$
\rho_{p}^{(N)}(M)=\operatorname{tr}_{a}\left(\left|\Psi^{(N)}(A)\right\rangle\left\langle\Psi^{(N)}(A)\right|\right),
$$

where the trace is with respect to the ancillary systems, and $\Psi$ is a MPS describing the spins and the ancillas.

Now we can apply the renormalization procedure for pure states to the tensor $A$, which induces a notion of RFP for the tensors corresponding to mixed states:

Definition 4.3. We call a tensor $M$ a Purification Renormalization Fixed Point (PRFP) if it can be written as (66), where $A$ is a RFP.

Note that the tensor $A$ in (65) will have the form (42), but now with two physical indices corresponding to the spin and the ancilla. When tracing over the ancilla indices, this will give rise to a superoperator, $\mathcal{E}$, which is a tpCPM. In particular, we can write

$$
\rho^{(N)}=\sum_{j, j^{\prime}=1}^{g} \sum_{q=1}^{r_{j}} \sum_{q^{\prime}=1}^{r_{j^{\prime}}} e^{i N\left(\phi_{j, q}-\phi_{j^{\prime}, q^{\prime}}\right)} \rho_{p}^{(N)}\left(A_{j}, A_{j^{\prime}}\right),
$$

where

$$
\rho_{p}^{(N)}\left(A_{j}, A_{j^{\prime}}\right)=\mathcal{E}^{\otimes N}\left[\left|\varphi_{j}\right\rangle\left\langle\left.\varphi_{j^{\prime}}\right|^{\otimes N}\right],\right.
$$

and $\varphi$ is given in (47). Note that any MPDO with a MPS purification can be written in the same way if $\mathcal{E}$ is asked to be only a CPM (not necessarily trace-preserving).

Thus, we can give the following characterization:

Theorem 4.4. The following statements are equivalent for a tensor $M$ fulfilling (66):

(i) $M$ is a PRFP

(ii) $M$ has $Z C L$

(iii) The density operators generated by $M$ have the form (67)

Even though it has a nice characterization in terms of zero-correlation length, Definition 4.3 has two main problems. The first one is that it only applies to MPDOs with a purification and, as shown in [33], this does not need to be the case. The second, and most important, is that it is too weak. As shown in Example 4.10 below, for mixed states ZCL does not immediately imply that there is no length scale in the system. In particular, it does not imply the saturation of the area law for the mutual information. This is different than for pure states, where the saturation of the area law is implied by ZCL (Proposition $3.14)$. 


\subsection{Mutual information. Saturation of the area law}

Throughout this Section, we will need to consider normalized states in order to talk meaningfully about entropic quantities. As a convention, whenever we consider one such entropic quantity for an MPDO $\rho^{(N)}(M)$, we will be referring to its normalized version

$$
\frac{\rho^{(N)}(M)}{\operatorname{tr}\left[\rho^{(N)}(M)\right]} .
$$

If we consider a chain with $N$ spins, the mutual information between a part of the chain of length $L$ and the rest is defined as

$$
I_{L}=S_{L}+S_{N-L}-S_{N}
$$

where $S_{L}$ is the von Neumann entropy of the reduced state of $L$ neighboring spins. For pure states, it coincides with (twice) the entanglement entropy of that part of the chain with the rest. It is not difficult to check (see Appendix C) that it fulfills the following monotonicity property

Proposition 4.5. For any $M P D O$ and $L<\lfloor N / 2\rfloor, I_{L} \leq I_{L+1}$ and

$$
\lim _{L \rightarrow \infty} \lim _{N \rightarrow \infty} I_{L}=I_{\infty}<\infty .
$$

This entails that the mutual information must increase and saturate with $L$, thus providing a length scale to the system. In a RFP one expects then a saturation of the area law as we define now

Definition 4.6. A tensor $M$ generating $M P D O$ fulfilling $I_{1}=I_{2}=\ldots$ is said to verify saturation of the area law $(S A L)$.

Below we will show examples of tensors which have ZCL but no SAL, nor the converse. In view of that, it makes sense to consider tensors with ZCL and SAL. According to their definition (4.6), they will have to fulfill $I_{L}=I_{L+1}$, or, equivalently, $S_{L}+S_{N-L}=S_{L+1}-S_{N-L-1}$ for $L<\lfloor N / 2\rfloor$. Note that this condition involves reduced density operators in which we have traced several spins. Thus, if the MPDO (51) contains terms that vanish whenever one traces few spins, then they will not be visible in the conditions we derive. More specifically, let us consider the tensor $M$ generating the MPDO as a MPV where the two indices (bra and ket) corresponding to the action on a spin are taken as physical indices. By assuming that we have blocked enough number of spins, we can write it in $\mathrm{CF}$ in terms of some BNT, $M_{k}$. Let us define

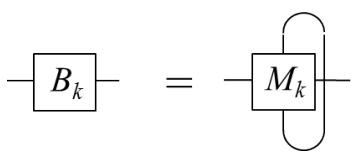

There may be some nilpotent $B_{k}$, meaning that $\operatorname{tr}_{R} \rho^{(N)}\left(M_{k}\right)=0$, where we have traced $R \leq D$ sites, and $N$ is sufficiently large. Thus, whenever we consider the entropy of a reduced state of the MPDO in which we have traced more than $D$ spins, we cannot state anything about the nilpotent terms in the BNT. This motivates the following: 
Definition 4.7. A tensor generating MPDO's is simple if none of the elements in a BNT is nilpotent.

We aim now to provide the equivalent of Theorem 3.10 for simple mixed states. In that theorem, three properties were shown to be equivalent for an MPS in CF: (i) being a RFP of some renormalization flow, characterized by the fact that there is a unitary (hence reversible) way to create one tensor out of two; (ii) the absence of some concrete type of length scales in the system, CID and LO in that case; (iii) its parent Hamiltonian being commuting.

The role of ZCL (equivalently CID and LO) will be played now by ZCL and SAL. While we have an analogue of (i) through the definition of RFP 4.1, we need to find an analogue of (iii). A natural choice, which also fits very well if one understands the given MPDO as the boundary theory of a 2D system (see Section 4.2), is the fact that the MPDO is of a special form.

Definition 4.8. We say that a density operator of $N$ spins is a Gibbs state of a nearest-neighbor commuting Hamiltionian (GSNNCH), if it can be written as

$$
\rho^{(N)} \propto \oplus_{x} n_{x} e^{-\sum_{j=1}^{N} \tau_{j}\left(h^{(x)}\right)} .
$$

where $n_{x}$ are natural numbers and the $h^{(x)}$ acts on the first two spins, $\tau_{j}$ translates the spins by an amount $j$, and $\left[h^{(x)}, \tau_{1}\left(h^{(x)}\right)\right]=0$.

The direct sum indicates that the global Hilbert space $\mathcal{H}=\oplus_{x} \mathcal{H}_{x}$, where $\mathcal{H}_{x}=\otimes_{n} \mathcal{H}_{n}^{x}$, and for each $n$, the $\mathcal{H}_{n}^{(x)}$ are orthogonal. The density operator (72) is translationally invariant, where the spin $N+1$ is identified with the first.

In the following we will establish the main result of this subsection. We will consider a simple tensor, $K$, in biCF, with $\mathrm{BNT} \mathcal{K}_{j}$ and corresponding coefficients $\mu_{j, q}$ [cf (20a)]. In order to emphasize that the tensor is simple, we will use $K$ instead of $M$, and $\sigma^{(N)}(K)$ instead of $\rho^{(N)}(K)$.

Theorem 4.9. Let us call $M$ the tensor obtained by blocking two sites:

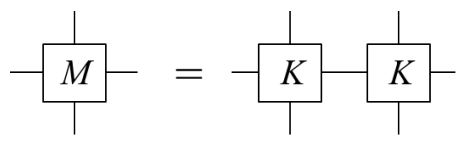

Consider the following statements:

(i) $K$ is a RFP.

(ii) $K$ has $Z C L$ and $S A L$.

(iii) The elements of a BNT are supported on different subspaces, i.e.

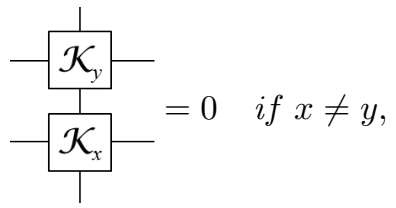


and can always be chosen such that $\sigma^{(N)}\left(\mathcal{K}_{j}\right) \geq 0$ for all $N$. Furthermore, for each BNT element, $\mathcal{K}$, there exists an isometry $U$ such that

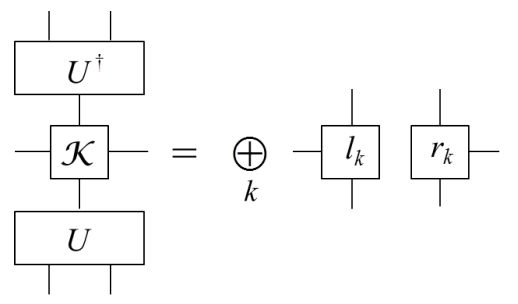

with

$$
\eta_{k, h}=\stackrel{+1}{r_{h}-\frac{1}{1}} \geq 0
$$

and

$$
\begin{aligned}
\operatorname{tr}\left(l_{k}\right) & =\mid \Phi) a_{k}, \\
\operatorname{tr}\left(r_{k}\right) & =b_{k}(\Psi \mid
\end{aligned}
$$

with

$$
\begin{aligned}
(\Psi \mid \Phi) & =1 \\
\sum_{k} a_{k} b_{k} & =1 .
\end{aligned}
$$

(iv) $\sigma^{(N)}(K)$ is a GSNNCH and $K$ has ZCL.

(v) $M$ is a RFP.

Then $i \Rightarrow i i \Leftrightarrow i i i \Leftrightarrow i v \Rightarrow v$.

The proof will be given in Appendix C. We finish this section giving two examples:

Example 4.10. (state with $Z C L$ but no $S A L$ ): Let us consider the state of four spins, each of them composed of two qubits. We identify by $(n, l)$ and $(n, r)$ the two qubits corresponding to the $n$-th spin (one to the left and the other to the right). Each qubit is maximally entangled with its neighbor. That is, $n r$ and $(n+1) l$ are in the state $(|0,0\rangle+|1,1\rangle) / \sqrt{2}$, where the spin 5 is identified with the first. We apply to each spin the tpCPM

$$
\mathcal{E}_{n}(X)=p \sigma_{x}^{(n, l)} \otimes \sigma_{x}^{(n, l)} X \sigma_{x}^{(n, l)} \otimes \sigma_{x}^{(n, l)}+(1-p) X .
$$

It is simple to show that $I_{2}>I_{1}$ for any $p \neq 0,1 / 2$. For instance, taking $p=0.25$, one can see that $S_{1}=2, S_{2}=2.9544, S_{3}=3.8802$, and $S_{4}=2.7839$, where $S_{i}$ is the entropy of $i$ neighboring spins. Thus, $I_{1}=3.0963$ and $I_{2}=$ 3.1250 and it does not saturate the area law. Furthermore, it has obviously ZCL since it fulfills (67). 
Example 4.11. (state with $S A L$ but no $Z C L$ ): We just have to take the same configuration as in the previous example, with $N$ spins, each of them composed of two qubits and define

$$
\sigma \propto \sum_{k_{1}, \ldots, k_{N}=0}^{1} \otimes_{n=1}^{N} \eta_{k_{n}, k_{n+1}}
$$

where $\eta_{k_{n}, k_{n+1}}$ acts on the qubits $(n, r)$ and $((n+1), l)$ (identifying $N+1$ with 1). We take

$$
\begin{aligned}
\eta_{0,0} & =|0\rangle\langle 0|\otimes| 0\rangle\langle 0|, \\
\eta_{0,1} & =\frac{1}{2}|0\rangle\langle 0|\otimes| 1\rangle\langle 1|, \\
\eta_{1,0} & =\frac{1}{2}|1\rangle\langle 1|\otimes| 0\rangle\langle 0|, \\
\eta_{1,1} & =|1\rangle\langle 1|\otimes| 1\rangle\langle 1|
\end{aligned}
$$

It is easy to show that this state fulfills the criterion of SAL. However, it does not have ZCL. A similar example for pure states also shows that in the case of MPS, saturation of the entanglement entropy does not suffice to characterize $R F P$.

\subsection{General case}

We have seen in the previous section that the RFP tensors generating MPDO can be fully characterized in terms SAL and ZCL for simple tensors. Those are the ones in which, when we trace one or more spins, we do not lose information about the tensor. Since, as shown in Theorem 4.9, the MPDO they generate are basically Gibbs states of commuting nearest neighbor Hamiltonians, they are, in some sense, trivial. For instance, they do not include the state considered in the following

Example 4.12. Let us consider a tensor, $M$, with $d=D=2$ and all components equal to zero except for: $1=M_{00}^{00}=M_{00}^{11}=M_{11}^{00}=-M_{11}^{11}$. The corresponding MPDO is

$$
\rho^{(N)}(M)=\mathbb{1}^{\otimes N}+\sigma_{z}^{\otimes N},
$$

where $\sigma_{z}=|0\rangle\langle 0|-| 1\rangle\langle 1|$. This is precisely the boundary state corresponding to the toric code [19, 26] (Section 4.2). If we trace one spin, the reduced state becomes proportional to the identity, i.e. one loses all the information about the $\sigma_{z}$ part. Note that this tensor is SAL and has ZCL. However, it cannot be expressed in the form given by (72). They nevertheless are RFP since one can easily find $\mathcal{S}$ and $\mathcal{T}$ [see (4.1)].

In this section we study RFP tensors that are general, and thus not necessarily simple. As we will see, their characterization will give rise to non-trivial 
solutions, which connect with unitary fusion categories and topologically ordered models in 2D.

In order to characterize the RFP property as defined in (55) for tensors $M$ generating MPDO, we have to use the $\mathrm{CF}$ of $M$ but in the vertical direction. That is, we will consider the tensor as generating MPV by concatenating it vertically, and thus by exchanging the notion of physical and virtual indices. We have

Proposition 4.13. A tensor $\tilde{M}$ in $C F$, generating $M P D O$, is also in $C F$ vertically. Moreover, there exists an isometry $U$ such that

$$
U \tilde{M} U^{\dagger}=\bigoplus_{\alpha} \mu_{\alpha} \otimes M_{\alpha}
$$

where $\mu_{\alpha}$ are diagonal and positive matrices, and $\left\{M_{\alpha}\right\}_{\alpha}$ is a BNT.

In graphical form, (83) simply means

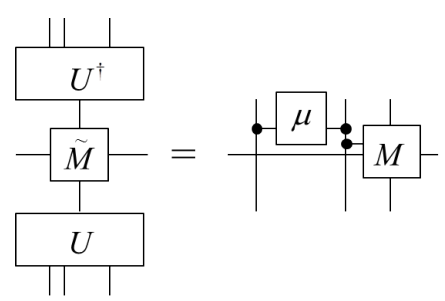

In what follows, we will ignore the isometry, as it just changes the physical basis on each site.

Given a tensor $M$ we define the operator $O_{L}(M)=\operatorname{tr}(M M \ldots M)$, where $M$ is multiplied $L$ times. Graphically, we have

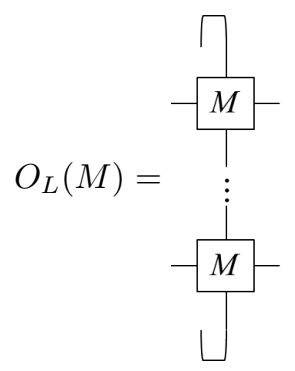

We also consider the corresponding operators for the BNT of $\mathrm{M}, O_{L}\left(M_{\alpha}\right)$.

Now, we can give the characterization of RFP, which is the main result of this paper.

Theorem 4.14. Given a tensor $M$ in $C F$ that generates $M P D O$, the following statements are equivalent:

(i) $M$ is a RFP. 
(ii) There exists a set of diagonal matrices, $\chi_{\alpha, \beta, \gamma}$, with positive elements, such that for each $L$ the operators $O_{L}\left(M_{\alpha}\right)$ linearly span an algebra with structure coefficients $c_{\alpha, \beta, \gamma}^{(L)}=\operatorname{tr}\left(\chi_{\alpha, \beta, \gamma}^{L}\right)$ :

$$
O_{L}\left(M_{\alpha}\right) O_{L}\left(M_{\beta}\right)=\sum_{\gamma} c_{\alpha, \beta, \gamma}^{(L)} O_{L}\left(M_{\gamma}\right)
$$

and

$$
m_{\gamma}=\sum_{\alpha, \beta} c_{\alpha, \beta, \gamma}^{(1)} m_{\alpha} m_{\beta}
$$

where $m_{\alpha}=\operatorname{tr}\left(\mu_{\alpha}\right)$. That is, the vector $\left(m_{\alpha}\right)_{\alpha}$ is an idempotent for the "multiplication" induced by $c^{(1)}$.

(iii) There exist isometries, $U_{\alpha, \beta}$, such that

$$
U_{\alpha, \beta} M_{\alpha} M_{\beta} U_{\alpha, \beta}^{\dagger}=\oplus_{\gamma} \chi_{\alpha, \beta, \gamma} \otimes M_{\gamma},
$$

where $\chi_{\alpha, \beta, \gamma}$ are the same as in the previous statement, and (87) is fulfilled.

This result is very appealing from the perspective of $2 \mathrm{D}$ topologically ordered models and boundary theories (Section 4.2), since it has been recently proven in [23] that starting from (86) and (87), for the particular case of $c_{\alpha, \beta, \gamma}^{(L)} \in \mathbb{N}$ and independent of $L$, one recovers in a direct and elegant way all known non-chiral topologically ordered phases together with a description of their excitations. The RFP MPDO we started with correspond exactly with the boundary theories of the RFP representatives of such topological phases [23]. This connection also gives immediately a large family of non-trivial RFP MPDO. We leave as an interesting open question whether there are more examples than those. More concretely, whether there exist RFP $M$ for which $c_{\alpha, \beta, \gamma}^{(L)}$ depends on $L$.

As showed in [23], the associativity of the multiplication in (86) imposes restrictions on the tensor $X=\left(\chi_{k, \alpha, \beta, \gamma}\right)_{\alpha, \beta, \gamma, k}$ that, by (88), give a pentagonlike equation for the $U_{\alpha, \beta}$. The solutions of such equation using F-symbols of unitary fusion categories give the family of RFP MPDO commented above: boundary theories of Levin-Wen string net models. In this sense, the topological content of a RFP MPDO lies in the unitaries $U_{\alpha, \beta}$ of (88).

Now, by applying equation (88) recursively, one may write any RFP MPDO given by a tensor $M$ as locally unitary equivalent -via the unitaries $U$ in (83)to $\left(\mu^{\otimes N}\right) \tilde{U} Q \tilde{U}^{\dagger}$, where $\mu=\sum_{\alpha} \mu_{\alpha} \otimes|\alpha\rangle\langle\alpha|$ is the tensor defined in (84); both $\mu^{\otimes N}$ and $\tilde{U} Q \tilde{U}^{\dagger}$ are completed by $\mathbb{1}$ in the spaces in which they do not act;

$$
\left[\mu^{\otimes N}, \tilde{U} Q \tilde{U}^{\dagger}\right]=0 ;
$$

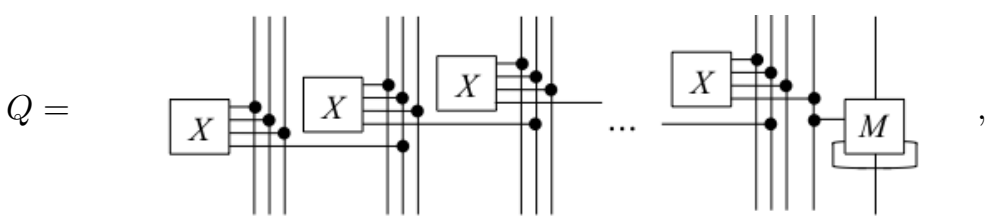


with each $X=\left(\chi_{\alpha, \beta, \gamma, k}\right)_{k, \alpha, \beta, \gamma}$ having assigned their indices in the order $k, \alpha, \beta, \gamma$; and $\tilde{U}$ is a sequential circuit of unitaries made out of the $U_{\alpha, \beta}$ 's in (88).

If the structure coefficients $c_{\alpha, \beta, \gamma}^{(L)}=\operatorname{tr}\left(\chi_{\alpha, \beta, \gamma}^{L}\right)$ are independent of $L$ one can easily show by Lemma A.5 that the $\chi_{\alpha, \beta, \gamma, k} \in\{0,1\}$ and therefore $c_{\alpha, \beta, \gamma}^{(L)} \in \mathbb{N}$. In this case, if the final matrices $\operatorname{tr}\left(M_{\gamma}\right)$ at the RHS of (90) are projectors, we have that $Q$ in (90) is also a projector. The same applies then to $\tilde{U} Q \tilde{U}^{\dagger}$. By making an spectral decomposition of the $\operatorname{tr}\left(M_{\gamma}\right)$ 's we can reduce to such case and obtain the following theorem.

Theorem 4.15. Start with a RFP tensor $M$ defining MPDO so that $c_{\alpha, \beta, \gamma}^{(L)}$ is independent of $L$. Then the MPDO

$$
\rho^{(N)}(M)=\sum_{i=1}^{d} \lambda_{i} P_{i}^{(N)} e^{-H_{N}}
$$

where $d$ is the single site Hilbert dimension, $P_{i}(N)$ are projectors which encode the topological properties of the tensor, if interpreted as a boundary theory, $H_{N}=\sum_{i=1}^{N} h_{i, i+1}$ is translationally invariant, nearest-neighbor and commuting $\left[h_{i-1, i}, h_{i, i+1}\right]=0$, and $\left[P_{i}, e^{-H}\right]=0$ for all $i$.

This result can be seen then as an analytical proof, for the case of RFP, of the conjecture stated in [26] about how boundary theories look like for topologically ordered models (Section 4.2).

\section{Conclusion}

In this paper we have given a full characterization of MPS and MPDO that are RFP in terms of their defining tensor. We have also shown that being a RFP can be also characterized by physical notions: zero-correlation length, saturation of the area law and being the ground or thermal state of commuting Hamiltionians.

The motivation of the paper, and arguably its main interest, lies on its connection with boundary theories and the classification program of $2 \mathrm{D}$ topological models on the lattice. It is believed that within each gapped topological quantum phase, there exists one representative that is the fixed point of some renormalization transformation. At a gapped RFP one expects an exact description of its ground state in terms of PEPS and an associated boundary theory which is a RFP MPDO. Here we have illustrated this for a concrete and natural definition of RFP in 2D but we expect the same result for any meaningful definition of RFP in 2D (we will analyze this in full detail in a forthcoming paper). Since all properties of the PEPS are encoded on its boundary via the boundary-bulk correspondence of [24], one can understand this paper as a way to classify 2D quantum phases on their boundary theories. From this perspective, our main result (Theorem 4.14) is very appealing, since it shows how the topological content of the bulk, at a RFP, can be clearly distilled from the canonical form of 
the MPDO that appears at the boundary. Moreover, when complemented with [23], our main result suggests that all quantum phases in 2D on the lattice seem to be already covered by Levin-Wen string net models and their generalizations.

JIC acknowledges support from the EU Integrated projects SIQS, and the DFG through the excellence cluster NIM. DPG acknowledges support from Comunidad de Madrid (grant QUITEMAD+-CM, ref. S2013/ICE-2801) and MINECO (grant no. MTM2014-54240-P). This project has received funding from the European Research Council (ERC) under the European Union's Horizon 2020 research and innovation program (grant agreement GAPS, No 648913). This work was made possible through the support of grant \#48322 from the John Templeton Foundation. The opinions expressed in this publication are those of the authors and do not necessarily reflect the views of the John Templeton Foundation. NS acknowledges funding by the European Research Council (ERC) under the European Union's Horizon 2020 research and innovation program (grant agreement WASCOSYS, No 636201). FV acknowledges funding by EU grant SIQS and ERC grant QUERG, the Odysseus grant from the Research Foundation Flanders (FWO) and the Austrian FWF SFB grants FoQuS and ViCoM. This research was supported in part by the National Science Foundation under Grant No. NSF PHY11-25915.

We thank Michael Mariën for very useful discussions. We also thank KITP (UC Santa Barbara), Perimeter Institute for Theoretical Physics and IQIM (Caltech) for their kind hospitality. This work was largely developed during the visits of the authors to those institutions.

\section{Appendix A. Proofs of Section 2}

To prove the Fundamental Theorem of MPV and the associated results stated in Section 2 we will need some lemmas that we will state and prove below. Before entering into that, and to connect also with previous (more strin-

gent) definitions of canonical forms for MPS, we will introduce the following definition:

Definition A.1. We say that a set of matrices $A^{i}$ are in a canonical form II (CFII), if they are in CF and the corresponding CPMs (15) are trace-preserving, and have a full-rank diagonal fixed point.

The two last properties can be expressed as

$$
\begin{aligned}
\sum_{i=1}^{d} A_{k}^{i \dagger} A_{k}^{i} & =\mathbb{1}_{D_{k}}, \\
\mathcal{E}_{k}\left(\Lambda_{k}\right) & =\sum_{i=1}^{d} A_{k}^{i} \Lambda_{k} A_{k}^{i \dagger}=\Lambda_{k}>0,
\end{aligned}
$$

where $\Lambda_{k}$ is a strictly positive definite diagonal $D_{k} \times D_{k}$ matrix. In $[28,27]$ it was shown that for any $A$ in $\mathrm{CF}$, it is always possible to find a non-singular 
gauge matrix $X$, such that

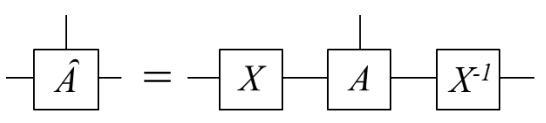

is also in CF and fulfills (A.1). For that one just has to determine the fixed point (i.e., the eigenvector corresponding to the largest eigenvalues in magnitude) of each $\mathrm{CPM} \mathcal{E}_{k}$.

Lemma A.2. Given two $N M P V, V_{a, b}$, generated by two $N T$ with corresponding $D_{\alpha} \times D_{\alpha}$ matrices $A_{\alpha}^{i}(\alpha=a, b)$, then

$$
\begin{aligned}
\lim _{N \rightarrow \infty}\left\langle V_{\alpha} \mid V_{\alpha}\right\rangle & =1, \\
\lim _{N \rightarrow \infty}\left|\left\langle V_{b} \mid V_{a}\right\rangle\right| & =0 \text { or } 1 .
\end{aligned}
$$

In the latter case, $D_{a}=D_{b}$ and there exists a non-singular matrix, $X$, and a phase $\phi$ so that $A_{b}^{i}=e^{i \phi} X A_{a}^{i} X^{-1}$.

Proof. Since $V_{a, b}$ are NMPV, the comment above shows that we can always find non-singular matrices, $Y_{a, b}$, such that

$$
\tilde{A}_{\alpha}^{i}=Y_{\alpha} A^{i} Y_{\alpha}^{-1}
$$

are in a CFII and generate the same MPV. We will use those matrices to prove the proposition. In particular, we will show that if $\left|\left\langle V_{b} \mid V_{a}\right\rangle\right| \rightarrow 1$ then there exists a unitary $X$ such that $X A_{b}^{i} X^{-1}=e^{i \phi} A_{a}^{i}$. The result for the original NT follows automatically from (A.4). First, $\left\langle V_{\alpha} \mid V_{\alpha}\right\rangle=\operatorname{tr}\left(\mathbb{E}_{\alpha}^{N}\right)$ where $\mathbb{E}_{\alpha}=$ $\sum_{i=1}^{d} A_{\alpha}^{i} \otimes \bar{A}_{\alpha}^{i}$. This is nothing but the matrix representation of the CPM built out of the matrices $A_{\alpha}^{i}$, and thus has the same spectrum. Since $A_{\alpha}$ is a NT, $\mathbb{E}_{\alpha}$ has a unique eigenvalue of magnitude 1 , and the rest are strictly smaller, from which follows that the norm tends to one for $N \rightarrow \infty$. Second, $\left\langle V_{b} \mid V_{a}\right\rangle=\operatorname{tr}\left(\mathbb{E}_{1,2}^{N}\right)$, where $\mathbb{E}_{a b}=\sum_{i=1}^{d} A_{a}^{i} \otimes \bar{A}_{b}^{i}$. Let us consider the eigenvalue equation for this matrix written as a linear map,

$$
\sum_{i=1}^{d} A_{b}^{i \dagger} X A_{a}^{i}=\lambda X
$$

Using the fact that the fixed point of $\mathcal{E}_{a}, \Lambda_{a}$, is strictly positive, we have

$$
\begin{aligned}
& \quad\left|\lambda \operatorname{tr}\left(X \Lambda_{a} X^{\dagger}\right)\right|^{2}=\left|\sum_{i=1}^{d} \operatorname{tr}\left(X A_{a}^{i} \sqrt{\Lambda_{a}} \sqrt{\Lambda_{a}} X^{\dagger} A_{b}^{i \dagger}\right)\right|^{2} \\
& \leq \sum_{i=1}^{d} \operatorname{tr}\left(A_{b}^{i} X \Lambda_{a} X^{\dagger} A_{b}^{i \dagger}\right) \sum_{j=1}^{d} \operatorname{tr}\left(X A_{a}^{j} \Lambda_{a} A_{a}^{j \dagger} X^{\dagger}\right) \\
& \leq\left|\operatorname{tr}\left(X \Lambda_{a} X^{\dagger}\right)\right|^{2}
\end{aligned}
$$


where we have used (A.1a,A.1b) and the Cauchy-Schwarz inequality. Since $\Lambda_{a}$ is full rank, $|\lambda|<1$ or $|\lambda|=1$, giving rise to 0 and 1 in (A.3b), respectively. Let us explore the latter case $|\lambda|=1$. This requires that the Cauchy-Schwarz if fulfilled with an equal sign, and since $\Lambda_{a}$ is non-singular,

$$
A_{b}^{i} X=\alpha X A_{a}^{i} .
$$

Using $X=\sum_{i=1}^{d} A_{b}^{i \dagger} A_{b}^{i} X=\alpha \sum_{i=1}^{d} A_{a}^{i \dagger} X A_{b}^{i}=\lambda \alpha X$, and thus $|\alpha|=1$. Then, $\sum_{i=1}^{d} A_{a}^{i \dagger} X^{\dagger} X A_{a}^{i}=X^{\dagger} X=\mathbb{1}$, and thus $X$ is an isometry. Furthermore, $X^{\dagger} A_{b}^{i} X=\alpha X^{\dagger} X A_{a}^{i}=\alpha A_{a}^{i}$. Let us assume that $D_{a}>D_{b}$; then there is a subspace of dimension $D_{a}-D_{b}$ such that $A_{a}^{i}$ vanishes on it, which is impossible given that $A_{a}$ is a NT. Thus, $D_{a}=D_{b}$, and $X$ is unitary.

From there we obtain the following two trivial corollaries:

Corollary A.3. Given two NT generating the $N M P V, V_{a, b}$, either $\left\langle V_{a} \mid V_{b}\right\rangle \rightarrow 0$ in the limit $N \rightarrow \infty$, or

$$
\left|V_{b}\right\rangle=e^{i \phi N}\left|V_{a}\right\rangle
$$

for all $N$.

Corollary A.4. Any set of NMPV, $\left\{V_{j}\right\}_{j=1}^{g}$, fulfilling $\left\langle V_{j} \mid V_{j^{\prime}}\right\rangle \rightarrow \delta_{j, j^{\prime}}$ for $N \rightarrow$ $\infty$, is linearly independent for $N$ sufficiently large.

We can now prove Proposition 2.7, that we restate here for the convenience of the reader

Proposition. The tensors $A_{j}(j=1, \ldots, g)$ form a BNT of $A$ iff: (i) for all $N T$ appearing in its $C F$ (17), $\tilde{A}_{k}$, there exists a $j$, a non-singular matrix $X_{k}$, and some phase $\phi_{k}$ such that

$$
\tilde{A}_{k}^{i}=e^{i \phi_{k}} X_{k} A_{j}^{i} X_{k}^{-1}
$$

(ii) the set is minimal, in the sense that for any element $A_{j}$, there is no other $j^{\prime}$ for which (A.8) is possible.

Proof. The result is an immediate consequence of the following procedure to construct a BNT for any given tensor $A$. First, express $A$ in CF. Let us denote by $\tilde{A}_{k}$ the NT appearing in the decomposition (17). Then, take $A_{1}:=\tilde{A}_{1}$. Second, if $\left\langle V^{(N)}\left(\tilde{A}_{2}\right) \mid V^{(N)}\left(A_{1}\right)\right\rangle \rightarrow 0$ as $N \rightarrow \infty$, take $A_{2}:=\tilde{A}_{2}$ and otherwise do not incorporate $\tilde{A}_{2}$ to the set, since according to Corollary A.3 $\left|V^{(N)}\left(\tilde{A}_{2}\right)\right\rangle=e^{i \phi_{2} N}\left|\tilde{V}^{(N)}\left(A_{1}\right)\right\rangle$. Then, continue in the same way; that is, if $\left\langle V^{(N)}\left(\tilde{A}_{k}\right) \mid \tilde{V}^{(N)}\left(A_{j}\right)\right\rangle \rightarrow 0$ for all $j=1, \ldots, J$, then $A_{J+1}:=\tilde{A}_{k}$. After this procedure, the BNT will be composed of a subset of $\tilde{A}_{k}$, say $A_{j}=\tilde{A}_{k_{j}}$, with $j=1, \ldots, g$. Note that if a NT, $\tilde{A}_{k}$, is not included in the BNT it must fulfill, according to Proposition A.2, $\left\langle V^{(N)}\left(\tilde{A}_{k}\right) \mid \tilde{V}^{(N)}\left(A_{j}\right)\right\rangle \rightarrow 1$ for some $j$, and thus there exist a non-singular matrix $X_{k}$, and some phase $\phi_{k}$ such that (A.8). 
Note that from Corollary A.3 it immediately follows that the elements of any two BNT corresponding to the same CF will coincide up to phases and a gauge transformation.

We need a final lemma, whose proof can be found e.g. in [33].

Let us consider two sets of complex numbers, $\lambda_{\alpha, k_{\alpha}}=\left|\lambda_{\alpha, k_{\alpha}}\right| e^{i \phi_{\alpha, k_{\alpha}}} \quad(\alpha=$ $\left.a, b, k_{\alpha}=1, \ldots, x_{\alpha}\right)$, sorted such that $\left|\lambda_{\alpha, k_{\alpha}}\right| \geq\left|\lambda_{\alpha, k_{\alpha}+1}\right|$ and $\phi_{\alpha, k_{\alpha}} \leq \phi_{\alpha, k_{\alpha}+1}$.

Lemma A.5. If $\forall N \leq \max \left\{x_{a}, x_{b}\right\}$,

$$
\sum_{k=1}^{x_{a}} \lambda_{a, k}^{N}=\sum_{k=1}^{x_{b}} \lambda_{b, k}^{N}
$$

then $x_{a}=x_{b}$ and $\lambda_{a, k}=\lambda_{b, k}$.

We can now prove the Fundamental Theorem of MPV, that we restate:

Theorem. Let $A$ and $B$ be two tensors in $C F$, with $B N T A_{k_{a}}^{i}$ and $B_{k_{b}}^{i}\left(k_{a, b}=\right.$ $\left.1, \ldots, g_{a, b}\right)$, respectively. If for all $N, A$ and $B$ generate $M P V$ that are proportional to each other, then: (i) $g_{a}=g_{b}=: g$; (ii) for all $k$ there exists a $j_{k}$, phases $\phi_{k}$, and non-singular matrices, $X_{k}$ such that $B_{k}^{i}=e^{i \phi_{k}} X_{k} A_{j_{k}}^{i} X_{k}^{-1}$.

Corollary. If two tensors $A$ and $B$ in $C F$ generate the same MPV for all $N$ then: (i) the dimensions of the matrices $A^{i}$ and $B^{i}$ coincide; (ii) there exists an invertible matrix, $X$, such that

$$
A^{i}=X B^{i} X^{-1} .
$$

Proof. To prove the Theorem we reason very similarly as in the proof of Proposition 2.7. Let us first consider $B_{k}$ for some given $k$. It is not possible that $\left\langle V^{(N)}\left(B_{k}\right) \mid V^{(N)}\left(A_{j}\right)\right\rangle \rightarrow 0$ as $N \rightarrow \infty$ for all $j$, since otherwise the MPV generated by $A$ and $B$ could not be proportional for all $N$ (Lemma A.4). Thus, according to Corollary A.3, there must exist one $j_{k}$ such that $\left|V^{(N)}\left(B_{1}\right)\right\rangle=$ $e^{i \phi_{k} N}\left|V^{(N)}\left(A_{j_{k}}\right)\right\rangle$. According to Lemma A.2 we have $B_{k}=e^{i \phi_{k}} X_{k} A_{j_{k}} X_{k}^{-1}$. We also conclude that $g_{a} \geq g_{b}$, But if we had considered $A_{k}$ to start with, we would obtain $g_{b} \geq g_{a}$, so that $g_{a}=g_{b}$.

To prove the Corollary, we denote by $A_{j}$ and $B_{j}$ the BNT which, according to the Theorem, have $g \mathrm{NT}$ and (after relabeling) $B_{j}=e^{i \phi_{j}} Y_{j} A_{j} Y_{j}^{-1}$. Denoting by $\mu_{j, q_{a}}$ and $\nu_{j, q_{b}}\left(j=1, \ldots, g, q_{x}=1, \ldots, r_{x, j}\right.$, with $\left.x=a, b\right)$ the coefficients in the decomposition of $A$ and $B$ in their BNT (20a), we must have

$$
\sum_{q=1}^{r_{a, j}} \mu_{j, q}^{N}=\sum_{q=1}^{r_{b, j}}\left(\nu_{j, q} e^{i \phi_{j}}\right)^{N}, \quad \forall j, N,
$$

From this equation it follows that $r_{a, j}=r_{b, j}=: r_{j}$ and $\mu_{j, q}=\nu_{j, q} e^{i \phi_{j}}$ (Lemma A.5). Then, the dimensions of the matrices $A^{i}$ and $B^{i}$ coincide. Also, defining

$$
Y=\oplus_{j=1}^{g} \mathbb{1}_{r_{j}} \otimes Y_{j}
$$

and using (20a), we have (A.10). 
We finish with the following

Corollary A.6. If in the above Theorem and Corollary the tensors are in CFII, the same applies but now with the $X$ and $X_{k}$ unitary.

\section{Appendix B. Proofs of Section 3}

Let us start proving Theorem 3.1. For that we start with the observation that the classes of tensors given by the relation (27) are in one to one correspondence with the set of CPM since two sets of matrices give the same CPM via the formula

$$
\mathcal{E}(X)=\sum_{i=1}^{d} A^{i} X A^{i \dagger}
$$

if and only if they are related by an isometry in the physical indices [34].

With this identification, it is easy to see that the renormalization step defined by blocking in Section 3 corresponds exactly to $\mathcal{E} \mapsto \mathcal{E}^{2}$ and, in particular, it does not depend on the representatives of the classes of tensors that are used. Moreover, if the renormalization flow has a limit, it must verify $\mathcal{E}=\mathcal{E}^{2}$. Using again that two sets of matrices giving the same CPM are related by an isometry in the physical indices we get Theorem 3.1. Using Theorem 3.8 (that we will prove below) and the fact that the transfer matrix $\mathbb{E}$ is the matrix associated to the linear map $\mathcal{E}$, this shows also the equivalence $(i) \Leftrightarrow(i i)$ in Theorem 3.10.

Let us now see that, if we start with a tensor $A$ in $\mathrm{CF}$, the renormalization procedure always converges. We write $A$ in terms of its BNT (20a). Given the block diagonal form of the matrices $A^{i}$, the transfer matrix is also block diagonal. In order to simplify the notation, we define

$$
\mathbb{E}^{\prime}=(X \otimes \bar{X}) \mathbb{E}(X \otimes \bar{X})^{-1},
$$

where $X$ is defined in (21). We have

$$
\mathbb{E}^{\prime}=\oplus_{j, j^{\prime}=1}^{g} \oplus_{q, q^{\prime}=1}^{r_{j}, r_{j^{\prime}}} \mu_{j, q} \bar{\mu}_{j^{\prime}, q^{\prime}} \mathbb{E}_{j, j^{\prime}},
$$

where

$$
\mathbb{E}_{j, j^{\prime}}=\sum_{i=1}^{d} A_{j}^{i} \otimes \bar{A}_{j^{\prime}}^{i}
$$

$\mathbb{E}_{j, j}$ can be considered as the matrix associated to the linear CPM (15). It thus has a single, nondegenerate, eigenvalue of magnitude 1 , with corresponding right and left eigenvectors $\left|R_{j}\right\rangle$ and $\left.\mid L_{j}\right)$, i.e.

$$
\begin{aligned}
\left.\mathbb{E}_{j, j} \mid R_{j}\right) & \left.=\mid R_{j}\right), \\
\left(L_{j} \mid \mathbb{E}_{j, j}\right. & =\left(L_{j} \mid,\right.
\end{aligned}
$$


If the tensor is in CFII, we have in addition

$$
\begin{aligned}
\left.\mid L_{j}\right) & \left.=\sum_{\alpha} \mid \alpha, \alpha\right), \\
\left.\mid R_{j}\right) & \left.=\sum_{\alpha}\left(\Lambda_{j}\right)_{\alpha, \alpha} \mid \alpha, \alpha\right),
\end{aligned}
$$

where $\Lambda_{j}>0$ and diagonal. The eigenvalues of $\mathbb{E}_{j, j^{\prime}}$ for $j \neq j^{\prime}$ must have a magnitude $<1$ (see Lemma A.2). The assumed normalization $\left(\left|\mu_{j q}\right| \leq 1\right.$ and at least one of them $=1$ ) implies then that the sequence $\mathbb{E}^{N}$, and hence the sequence $\mathcal{E}^{N}$, will converge always.

Let us move now to prove Theorem 3.8, that is, ZCL (defined as CID and LO) is equivalent to the property $\mathbb{E}^{2}=\mathbb{E}$ for a tensor $A$ in $\mathrm{CF}$. The above argument shows such a tensor $A$ is LO if and only if $\mathbb{E}_{j, j^{\prime}}=0$ for all $j \neq j^{\prime}$. Let's assume then that property and prove that then $\mathbb{E}^{2}=\mathbb{E}$ is equivalent to CID. This will finish the proof of Theorem 3.8. Wlog we will assume for simplicity that we work with $\mathbb{E}^{\prime}$ as defined in (B.2).

Clearly, if $\mathbb{E}^{2}=\mathbb{E}$, equation (39) shows that $A$ has CID. For the converse we reason as follows. Assume that $\mathbb{E}^{2} \neq \mathbb{E}$. This implies that there exists a NT in $A$, wlog $A_{1}$, so that $\mathbb{E}_{1}^{2} \neq \mathbb{E}_{1}$ and hence there is a non-zero eigenvalue $\lambda$ smaller than 1. Call $\left.\left.\mid r_{1}\right), \mid l_{1}\right)$ to the associated right and left eigenvectors (so that $\left.\left(l_{1} \mid r_{1}\right)=1\right)$. The block-injective property guaranteed by Proposition 2.9 ensures the existence of operators $O_{1}, O_{2}$, supported on regions of size $3 D^{5}$ so that

$$
\mathbb{E}_{O_{1}}=P_{j=j^{\prime}=1}\left(\oplus_{q, q^{\prime}=1}^{r_{1}}\left(\mu_{1, q} \bar{\mu}_{1, q^{\prime}}\right)^{3 D^{5}} \mid R_{1}\right)\left(l_{1} \mid\right) P_{j=j^{\prime}=1}
$$

and

$$
\mathbb{E}_{O_{2}}=P_{j=j^{\prime}=1}\left(\oplus_{q, q^{\prime}=1}^{r_{1}}\left(\mu_{1, q} \bar{\mu}_{1, q^{\prime}}\right)^{3 D^{5}} \mid r_{1}\right)\left(L_{1} \mid\right) P_{j=j^{\prime}=1}
$$

where $P_{j=j^{\prime}=1}$ projects on the sector $j=j^{\prime}=1$ of the decomposition (B.3).

Due to formula (39), the correlation function

$$
\left\langle V^{(N)}(A)\left|O_{1} O_{2}\right| V^{(N)}(A)\right\rangle=\lambda^{\operatorname{dist}\left(O_{1}, O_{2}\right)} \sum_{q, q^{\prime}=1}^{r_{1}}\left(\mu_{1, q} \bar{\mu}_{1, q^{\prime}}\right)^{N}
$$

Recall that by Lemma A.5,

$$
\sum_{q, q^{\prime}=1}^{r_{1}}\left(\mu_{1, q} \bar{\mu}_{1, q^{\prime}}\right)^{N}=\left|\sum_{q=1}^{r_{1}} \mu_{1, q}^{N}\right|^{2} \neq 0
$$

for infinitely many $N$ s. Hence the tensor does not have CID, what finishes the proof of Theorem 3.8.

The next step is to prove Theorem 3.11, giving the structural characterization for RFP tensors in CF presented in (42). For that we need the following lemma. 
Lemma B.1. A NT A is a RFP iff it can be written as

$$
A^{i}=X \Lambda U^{i} X^{-1},
$$

where $\Lambda$ is diagonal, positive, with $\operatorname{tr}(\Lambda)=1$, and $U$ is an isometry

$$
\sum_{i=1}^{s} U_{\alpha, \beta}^{i} \bar{U}_{\alpha^{\prime}, \beta^{\prime}}^{i}=\delta_{\alpha, \alpha^{\prime}} \delta_{\beta, \beta^{\prime}}
$$

We can express graphically (B.11) as follows

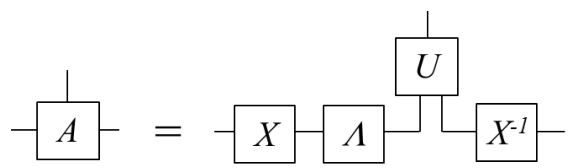

Proof. Let us take a NT $A$ which is a RFP (the reverse implication is trivial). Let us first assume that $A$ is in CFII. Since $A$ is a NT, its transfer matrix has a single block. Moreover, being a $\mathrm{RFP}, \mathbb{E}^{2}=\mathbb{E}$, which implies that $\left.\mathbb{E}=\mid R\right)(L \mid$ with $(L \mid R)=1$. Moreover,

$$
\left.\mid R)=\sum_{\alpha=1}^{D} \Lambda_{\alpha} \mid \alpha, \alpha\right), \quad\left(L \mid=\sum_{\alpha=1}^{D}(\alpha, \alpha \mid\right.
$$

This transfer matrix is obtained by a tensor whose physical index is a doubleindex, $i=\left(\alpha^{\prime}, \beta^{\prime}\right), A_{\alpha, \beta}^{\left(\alpha^{\prime}, \beta^{\prime}\right)}=\delta_{\alpha, \alpha^{\prime}} \delta_{\beta, \beta^{\prime}} \sqrt{\Lambda_{\alpha}}$. Any other tensor giving rise to the same transfer matrix must be related to this one by an isometry $U$ acting on the physical indices. Thus, we arrive at (B.11) with $X=\mathbb{1}$. The appearance of $X$ follows from the fact that $A$ is in $\mathrm{CF}$, and thus it is related by a similarity transformation to its CFII.

The proof of Theorem 3.11 follows immediately now if we use the decomposition of $A$ in BNT elements (20a), and the form of each NT in the RFP given in the previous proposition. Note that the proof of RFP $\Leftrightarrow$ ZCL shown above gives explicitly that if $A$ is a RFP, each one of the elements in the BNT is a RFP too.

Let us finish this section showing the remaining equivalence in Theorem 3.10. Namely that RFP $\Leftrightarrow$ NNCPH.

The implication RFP $\Rightarrow \mathrm{NNCPH}$ is trivial from Theorem 3.11. To prove the reverse, we will use [35] where it is shown that the ground space of any nearestneighbor commuting Hamiltonian in a 1D spin chain with a finite (independent of system size) degeneracy $g$ is spanned by $g$ states of the form (46) that are locally orthogonal. By the defining properties of the parent Hamiltonian, they must be then a BNT for the original tensor $A$, and they are RFP. Tensor $A$ has then the form given in (42) and is a RFP by Theorem 3.11. 


\section{Appendix C. Proofs of Section 4}

\section{Appendix C.1. Proof of Proposition 4.5}

Proof. We show here that for a translationally invariant MPDO of $N$ spins and finite bond dimension, $D$, the mutual information, $I_{L}$, is a monotonically increasing function $\left(I_{L} \leq I_{L+1}\right)$ for $L<\lfloor N / 2\rfloor$, and that it is bounded by a constant that only depends on $D$. The latter immediately follows from the fact that for any MPDO the mutual information fulfills an area law, $I_{L} \leq 4 \log D$, as proven in [36]. In order to prove monotonicity, we divide the spin chain in four consecutive segments, $A, B, C$ and $D$, consisting of $N-2 L-1, L, 1$ and $L$ spins, respectively (note that $N-2 L-1>0$ since $L<\lfloor N / 2\rfloor$ ). We use the strong subaddivity inequality for the entropy, $S_{B}+S_{A B C} \leq S_{A B}+S_{B C}$, where $S_{X}$ denotes the entropy of the reduce state in region $X$. Substracting $S_{A B C D}=S_{N}$ on both sides of the inequality, we obtain the desired monotonicity condition.

\section{Appendix C.2. Proof of Theorem 4.9}

In this subsection we will slightly change the graphical representation, due to the fact that in some instances we will have to consider tensors with many indices. We will follow the convention that in tensors generating MPDO we will represent the bra indices with dashed lines whenever we write them on the other side of the tensors. For instance, the tensor $K$ generating the MPDO $\sigma^{(N)}(K)$ will be interchangeably written as

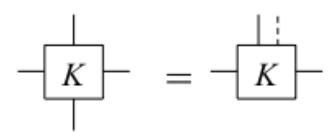

We start out with the first implication of Theorem 4.9, which is the simplest one:

Proposition C.1. If a tensor $K$ is a RFP then it satisfies $Z C L$ and $S A L$.

Proof. Since $K$ is a RFP, there exist two tpCMP, $\mathcal{T}$ and $\mathcal{S}$, fulfilling (55). The fact that it has ZCL is a direct consequence of (54) and that $\mathcal{T}$ preserves the trace: $\operatorname{tr}\left[K_{2}(X)\right]=\operatorname{tr}\left[\mathcal{T}\left[K_{1}(X)\right]\right]=\operatorname{tr}\left[K_{1}(X)\right]$ for all $X$, and thus $K$ has ZCL according to Definition 4.2. In order to prove SAL, let us take the chain, and remind that according to Proposition 4.5, for $L<\lfloor N / 2\rfloor$ we have that $I_{L} \leq I_{L+1}$. In order to prove the equality, we separate the chain into two regions, $\mathrm{A}$ and $\mathrm{B}$, of size $L$ and $N-L$, respectively, with $L<\lfloor N / 2\rfloor$, so that the mutual information between $\mathrm{A}$ and $\mathrm{B}$ is $I_{L}$. Now we apply $\mathcal{T}$ to the first spin of region $\mathrm{A}$, and $\mathcal{S}$ to the first two spins of region $\mathrm{B}$, so that the mutual information between $\mathrm{A}$ and $\mathrm{B}$ will become $I_{L+1}$. Given that the mutual information cannot increase by local operations, we have that $I_{L+1} \leq I_{L}$, which completes the proof. 
We continue now with a simple property that follows from SAL. Let us define $\sigma_{3}^{(N)}$ as the state that is obtained from $\sigma^{(N)}(K)$ by tracing all the spins but the first three. Graphically

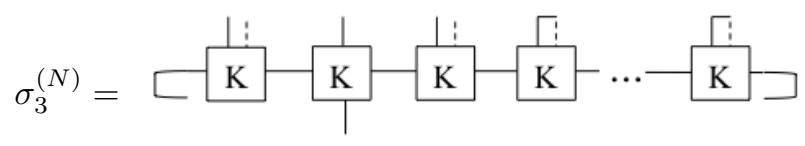

Let us call those spins $A, B$, and $c$, and the corresponding Hilbert spaces, $H_{A}, H_{B}$, and $H_{c}$, respectively. Then we have

Lemma C.2. If the tensor $K$ has $S A L$, then we can decompose $H_{B}=\oplus_{k} H_{b_{1}}^{(k)} \otimes$ $H_{b_{2}}^{(k)}$, so that

$$
\sigma_{3}^{(N)}=\oplus_{k} \rho_{A b_{1}}^{(k)} \otimes \rho_{b_{2} c}^{(k)}
$$

where $\rho_{A b_{1}}^{(k)}$ is supported on $H_{A} \otimes H_{b_{1}}^{(k)}$ and $\rho_{b_{2} c}^{(k)}$ on $H_{b_{2}}^{(k)} \otimes H_{c}$.

Proof. We divide the whole spin chain composed of $N$ spins into four connected regions, $A, B, C$, and $D$, with $1,1, N-3$, and 1 spin, respectively. SAL implies that $I_{2}=I_{1}$, which is equivalent to $S_{A B C}+S_{B}=S_{A B}+S_{B C}$, i.e. strong subadditivity is fulfilled as an equality. In this case, we can employ the characterization of density operators satisfying this equality [40]: we first divide the Hilbert space $H_{A B C}=\oplus_{k} H_{A b_{1}}^{(k)} \otimes H_{b_{2} C}^{(k)}$, with $H_{A b_{1}}^{(k)}=H_{A} \otimes H_{b_{1}}^{(k)}\left(H_{b_{2} C}^{(k)}=H_{b_{2}}^{(k)} \otimes H_{C}\right)$. Then, we write the reduced state $\sigma_{A B C}=\oplus_{k} \rho_{A b_{1}}^{(k)} \otimes \rho_{b_{2} C}^{(k)}=\sigma_{N-1}^{(N)}$, where the direct sum is with respect to region $B$, and $\rho_{A b_{1}}^{(k)}\left(\rho_{b_{2} C}^{(k)}\right)$ is supported on $H_{A b_{1}}^{(k)}$ $\left(H_{b_{2} C}^{(k)}\right)$. The operator $\sigma_{N-1}^{(N)}$ is nothing but the original one, $\sigma^{(N)}(\mathcal{K})$, where we have traced the last spin. Now, if we trace all the spins in region $C$ but the first one, we obtain (C.3).

Definition C.3. We will denote by $Q_{k}$ the projector onto $H_{b_{1}}^{(k)} \otimes H_{b_{2}}^{(k)}$.

Note that in the proof of Lemma C.2, the direct sum structure is derived for $\sigma_{N-1}^{(N)}$ and not for $\sigma^{(N)}$. That is, we always trace at least one spin from the chain. This is why in the whole Section 4.4 we have to deal with simple tensors, i.e. that when we trace a spin none of the elements of a BNT are eliminated.

Appendix C.2.1. Case I: $K$ injective

In this subsection we will assume $K$ to be an injective NT generating MPDOs. In order to emphasize this fact, we will denote the tensor by $\mathcal{K}$. The fact that it is injective implies that [compare (25)]

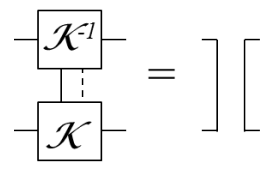


which will be used in some of the proofs. In the next subsection we will relax this condition and consider general simple tensors.

One of the main purposes of this subsection is to relate SAL and ZCL with the existence of an isometry, $U$, and tensors $r_{k}, l_{k}$, such that [compare Proposition 4.9]

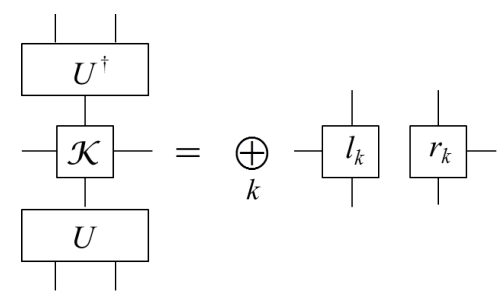

with

$$
\eta_{k, h}=\stackrel{+}{r_{h}}-\frac{1}{l_{k}} \geq 0
$$

and

$$
\begin{aligned}
\operatorname{tr}\left(l_{k}\right) & =\mid \Phi) a_{k}, \\
\operatorname{tr}\left(r_{k}\right) & =b_{k}(\Psi \mid
\end{aligned}
$$

with

$$
\begin{aligned}
(\Psi \mid \Phi) & =1 \\
\sum_{k} a_{k} b_{k} & =1 .
\end{aligned}
$$

Let us recall that a non-negative matrix -i.e. a matrix with non-negative elements-is called primitive [37] if there exist some finite $n_{0}$ such that $\left\langle k\left|T^{n_{0}}\right| h\right\rangle>$ 0 for all $k, h$. For a primitive matrix, $\lim _{n \rightarrow \infty}\left(T^{n}\right)_{k, h}=a_{k} b_{h}$ for some real numbers $a_{k}$ and $b_{h}$.

Lemma C.4. If $\mathcal{K}$ fulfills $S A L$, there exists an isometry, $U$, and tensors $r_{k}, l_{k}$, such that (C.5), with (C.6), and the non-negative matrix defined by

$$
T_{k, h}=\operatorname{tr}\left(\eta_{k, h}\right)
$$

is primitive.

Proof. Since $\mathcal{K}$ fulfills SAL, we can use the characterization of Lemma C.2. Now, we project the second spin using $Q_{k}$, and apply the map $\mathcal{K}^{-1}$ to the first and third spin of $\sigma_{3}^{(N)}$. We denote by

$$
X_{\alpha, \beta}=\alpha-\frac{1}{\mathcal{K}^{1}-\beta}
$$

and

$$
Q_{k} \operatorname{tr}_{1,3}\left[\left(X_{\alpha_{1}, \beta_{1}} \otimes \mathbb{1}_{2} \otimes X_{\alpha_{3}, \beta_{3}}\right) \sigma_{3}^{(N)}\right] Q_{k}=A_{\alpha_{1}, \beta_{1}}^{(k)} \otimes B_{\alpha_{3}, \beta_{3}}^{(k)}
$$


where $A_{\alpha_{1}, \beta_{1}}^{(k)}=\operatorname{tr}_{1}\left(X_{\alpha_{1}, \beta_{1}} \rho_{A b_{1}}^{(k)}\right)$ and similarly with $B_{\alpha_{3}, \beta_{3}}^{(k)}$. Taking (C.2) for $\sigma_{3}^{(N)}$, and using (C.4) in the computation of the lhs of (C.11) we get

$$
A_{\alpha_{1}, \beta_{1}}^{(k)} \otimes B_{\alpha_{3}, \beta_{3}}^{(k)}=m_{\beta_{3}, \alpha_{3}} \quad \beta_{1}-\stackrel{\mathcal{K}}{\perp}-\alpha_{3}
$$

with

$$
m_{\beta, \alpha}=\beta-\bar{K}-\ldots \stackrel{\Gamma}{\mathcal{K}}-\alpha
$$

It is clear that we can always choose $\alpha$ and $\beta$ such that $m_{\beta, \alpha} \neq 0$ [since otherwise $\sigma^{N-3}(\mathcal{K})$ would vanish]. Thus we can write

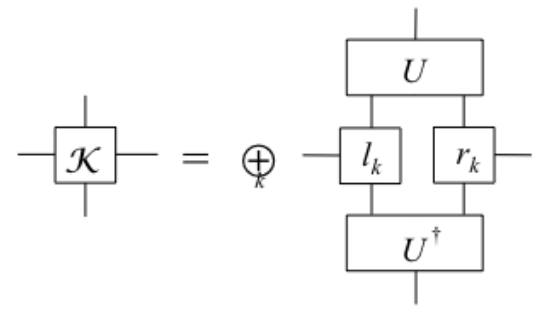

for some tensors $r_{k}$ and $l_{k}$. The direct sum structure refers to the physical (vertical) indices, as it is inherited from the splitting of the space $H_{B}$ defined above. The isometry $U$ just changes the local basis such that in each subspace defined by $Q_{k}$, we have the tensor product structure $H_{b_{1}}^{(k)} \otimes H_{b_{2}}^{(k)}$. In the following, we will work in that basis, by defining $\tilde{\sigma}=U^{\dagger \otimes N} \sigma^{(N)}(\mathcal{K}) U^{\otimes N} \geq 0$.

We proceed by defining

$$
\eta_{k, h}=\stackrel{+}{r_{k}} l_{h}^{+1}
$$

Then, according to (C.14) we have

$$
0 \leq\left[Q_{k_{1}} \otimes \ldots Q_{k_{N}}\right] \tilde{\sigma}\left[Q_{k_{1}} \otimes \ldots Q_{k_{N}}\right]=\otimes_{n=1}^{N} \eta_{k_{n}, k_{n+1}}
$$

with $k_{N+1}=k_{1}$. It is then clear that the $\eta$ 's can be chosen positive semidefinite.

We define now

$$
T_{k, h}=\operatorname{tr}\left(\eta_{k, h}\right) \geq 0,
$$

and thus it is a non-negative matrix. We can always find some finite integer $n$ such that we can write $T^{n}=\oplus_{x} T^{(x)}$, where $T^{(x)}$ is primitive for each $x$ [37] (since by blocking we can get rid of the $p$-periodic components, as it is in the case of CPMs; see discussion after Eq.(15)). For simplicity, we will assume that $n=1$, but the proof can be easily generalized to an arbitrary $n$. Thus, we can 
split the different $k$ in disjoint sets, $S_{x}$, so that $\eta_{k, k^{\prime}}=0$ if $k$ is in a different set than $k^{\prime}$. Thus, we can write

$$
\tilde{\sigma}=\oplus_{x} \tilde{\sigma}_{x}
$$

where each

$$
\tilde{\sigma}_{x}=\oplus_{k_{1}, \ldots, k_{N} \in S_{x}} \otimes_{n=1}^{N} \eta_{k_{n}, k_{n+1}}
$$

The tensors generating each $\tilde{\sigma}_{x}$ must be locally orthogonal (see Definition 3.5 ), since they correspond to different $k$ 's. This is incompatible with the fact that $\mathcal{K}$ is injective, unless there is a single $x$, i.e. $T$ is primitive. This last statement can be proven as follows: assume that there are two values of $x=1,2$. Then we can find two orthogonal projectors, $P_{1,2}$ with $P_{1}^{(1)} \tilde{\sigma} P_{2}^{(1)}=P_{2}^{(1)} \tilde{\sigma} P_{1}^{(1)}=$ $\left(P_{1}^{(1)} \otimes P_{2}^{(2)}\right) \tilde{\sigma}\left(P_{1}^{(1)} \otimes P_{2}^{(2)}\right)=0$, where the superscript indicates on which spin they are acting on. Since $\mathcal{K}$ is injective, we can use (C.4) to obtain

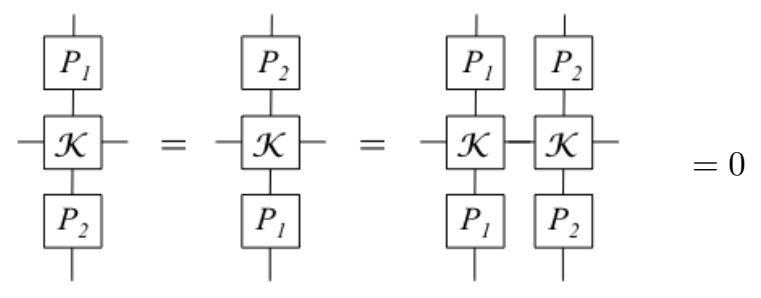

It is now relatively easy to show that this is incompatible with the fact that $\mathcal{K}$ is injective. For that, let us denote by $\mathcal{A}_{1,2}$ the linear spaces of matrices generated by $\operatorname{tr}\left(X P_{1,2} \mathcal{K} P_{1,2}\right)$, respectively, for all $X$. According to the first two conditions in (C.20), the linear space of matrices generated by $\operatorname{tr}(X \mathcal{K}), \mathcal{A}$, is $\mathcal{A}_{1}+\mathcal{A}_{2}$. Furthermore, the last condition in (C.20) implies that $\mathcal{A}_{1} \mathcal{A}_{2}=0$, and thus $\mathcal{A}$ cannot be the whole set of matrices, from which we conclude that $\mathcal{K}$ cannot be injective. This statement also holds if there are more than two values of $x$.

So far we have not introduced the condition of ZCL. It will restrict the properties of the tensors $r_{k}$ and $l_{k}$ defined in (C.14). For the next proposition we need to define

$$
\begin{gathered}
\left.\frac{\Gamma}{l_{k}}=\mid l_{k}\right) \\
r_{k}=\left(r_{k} \mid\right.
\end{gathered}
$$

where the tensors are the ones used to build up the $\eta$ 's in (C.15). Note that

$$
T_{k h}=\left(r_{k} \mid l_{h}\right)
$$

Lemma C.5. If $\mathcal{K}$ fulfills both $S A L$ and $Z C L$, then there exist two vectors, $\Phi$, and $\Psi$ and real numbers, $a_{k}, b_{k}$, such that (C.7) and (C.8). 
Proof. According to Definition 4.2 and (C.22), ZCL implies

$$
\left.\sum_{k} \mid l_{k}\right)\left(r_{k}\left|=\sum_{k, h} T_{k, h}\right| l_{k}\right)\left(r_{h} \mid\right.
$$

Thus,

$$
\operatorname{tr}\left(T^{N}\right)=\operatorname{tr}(T)=\sum_{k}\left(r_{k} \mid l_{k}\right)
$$

for all $N$. Using the theory of fixed points of non-negative matrices [37], and taking into account that $T$ is primitive (see Lemma C.4), this in turn implies that $T$ has one eigenvalue equal to one and the rest are zero, so that $T_{k, h}=a_{k} b_{h}$, where $a_{k}, b_{h}$ are real numbers and that $\operatorname{tr}(T)=1$, which results in $(\mathrm{C} .8 \mathrm{~b})$. We also conclude that

$$
\left.\sum_{k} \mid l_{k}\right)\left(r_{k} \mid=\left[\sum_{k} a_{k} \mid l_{k}\right)\right]\left[\sum_{h} b_{h}\left(l_{k} \mid\right] \propto \mid \Phi\right)(\Psi \mid
$$

where we have chosen $(\Phi \mid \Psi)=1$. Then each of the terms in the sum has to be also proportional to $\mid \Phi)\left(\Psi \mid\right.$, so that we have (C.7). Since $T_{k, h}=\left(l_{k} \mid r_{h}\right)=a_{k} b_{h}$, we have the rest of the statements of the lemma.

Putting together lemmas C.4 and C.5, we have

Corollary C.6. If $\mathcal{K}$ fulfills both $S A L$ and $Z C L$, there exists an isometry, $U$, tensors $r_{k}$ and $l_{k}$, vectors $\Phi$ and $\Psi$, and real numbers $a_{k}, b_{k}$ such that (C.5), with (C.6), (C.7), and (C.8).

This last corollary gives us the structure of the tensors fulfilling both SAL and ZCL. Now we characterize the MPDO generated by those tensors. We will show that they are GSNNCG (see Definition 72).

Proposition C.7. The MPDO generated by a $\mathcal{K}$, fulfilling (C.5), (C.6), (C.7), and (C.8), has the form

$$
\sigma^{(N)}(\mathcal{K}) \propto e^{-\sum_{n=1}^{N} h_{n, n+1}},
$$

where $h_{n, n+1}$ act on nearest neighbors and commute, i.e. $\left[h_{n-1, n}, h_{n, n+1}\right]=0$; furthermore, $\mathcal{K}$ has $Z C L$.

Proof. We define

$$
\tilde{\sigma}=U^{\dagger \otimes N} \sigma^{(N)}(\mathcal{K}) U^{\otimes N}
$$

Using (75) we have

$$
\tilde{\sigma}=\oplus_{k_{1}, \ldots, k_{N}}\left[\otimes_{n=1}^{N} \eta_{k_{n}, k_{n+1}}\right]
$$

Here, the physical Hilbert space of each spin is decomposed as $H_{n}=\oplus_{k} H_{L, n}^{(k)} \otimes$ $H_{R, n}^{(k)}$ and $\eta_{k_{n}, k_{n+1}} \geq 0$ is acting on $H_{R, n}^{\left(k_{n}\right)} \otimes H_{L, n+1}^{\left(k_{n+1}\right)}$. If we trace the second spin in $\eta_{k, h}$,

$$
\left.\operatorname{tr}_{2}\left(\eta_{k, h}\right)=\underset{r_{k}}{r_{1}}-\Gamma_{l_{h}}=a_{h} \underset{r_{k}}{r_{1}}-\mid \Phi\right)
$$


so that the range of this trace is independent of the value of $h$. The same thing applies if we trace the first spin. This implies that $\eta_{k, h}$ has full rank in $H_{R, n}^{(k)} \otimes H_{L, n+1}^{(h)}$. Thus, we can take the logarithm of $\eta_{k, h}$, and define it as $-\tilde{h}_{k, h}$. Therefore, we can write [compare (C.28)]

$$
\tilde{\sigma}=\bigoplus_{k_{1}, \ldots, k_{N}} e^{\left.-\sum_{n=1}^{N} \tilde{h}_{n, n+1}^{\left(k_{n}, k_{n}+1\right.}\right)}
$$

where $\tilde{h}_{n, n+1}^{\left(k_{n}, k_{n+1}\right)}$ acts on the $n$-th and $n+1$-th spins. By defining $h_{n, n+1}=$ $\sum_{k_{n}, k_{n+1}} \tilde{h}_{n, n+1}^{\left(k_{n}, k_{n+1}\right)}$ it is a simple exercise to show that this expression can indeed be written as (C.26), where the isometry $U$ is included in the definition of the $h$ 's. It is also straightforward that the $h$ commute with each other. Furthermore, (C.6), (C.7), and (C.8) immediately imply that $K$ has ZCL, since

$$
\left.-\stackrel{\Gamma}{\mathcal{K}}=\sum_{k} a_{k} b_{k} \mid \Phi\right)(\Psi|=| \Phi)(\Psi \mid
$$

where we have used (C.8b). This matrix has rank one according to (C.8a) and is thus idempotent.

Now we show that:

Proposition C.8. If $\mathcal{K}$ generates MPDOs of the form (C.26) then it fulfills $S A L$.

Proof. Let us divide the chain into four regions, A, B, C, and D, containing $m-1,1, N-m-1$, and 1 subsequent spins, respectively, and by $H_{X}(X=$ $A, B, C, D)$ the corresponding Hilbert spaces. Since the Hamiltonians $h$ commute, we can use the characterization of [35, 39]; we divide $H_{B}=\oplus_{k} H_{b_{1}}^{(k)} \otimes H_{b_{2}}^{(k)}$, and define $R_{k}$ as the projector onto the subspace labeled by $k$. We thus have

$$
\begin{aligned}
h_{m-1, m} & =\sum_{k} \tilde{h}_{A b_{1}}^{(k)} \\
h_{m, m+1} & =\sum_{k} \tilde{h}_{b_{2} C}^{(k)} \\
\sum_{n=1}^{m-2} h_{n, n+1} & =\tilde{h}_{A} \\
\sum_{n=m+1}^{N} h_{n, n+1} & =\tilde{h}_{C}
\end{aligned}
$$

where $\tilde{h}_{A b_{1}}^{(k)}$ acts on $H_{A} \otimes H_{b_{1}}^{(k)}, \tilde{h}_{b_{2} C}^{(k)}$ on $H_{b_{2}}^{(k)} \otimes H_{C}, \tilde{h}_{A}$ on $H_{A}$ and $\tilde{h}_{C}$ on $H_{C} \otimes H_{D}$. Denoting further

$$
\begin{aligned}
& \rho_{A b_{1}}^{(k)}=R_{k} e^{-\tilde{h}_{A}-\tilde{h}_{A b_{1}}^{(k)} R_{k}} \\
& \rho_{b_{2} C}^{(k)}=R_{k} e^{-\tilde{h}_{C}-\tilde{h}_{b_{2} C}^{(k)}} R_{k}
\end{aligned}
$$


we can rewrite $(\mathrm{C} .26)$ as

$$
e^{-\sum_{n=1}^{N} h_{n, n+1}}=\sum_{k} R_{k} e^{-\sum_{n=1}^{N} h_{n, n+1}}=\oplus_{k} \rho_{A b_{1}}^{(k)} \otimes \rho_{b_{2} C}^{(k)}
$$

where $R_{k}$ is acting on the spin in region $B$, and commutes with all the terms. Thus, the MPDO precisely adopts the form which saturates the strong subadditivity inequality between regions $\mathrm{A}, \mathrm{B}$, and C [40], i.e. $S_{A B C}+S_{B}=S_{A B}+S_{B C}$, which immediately implies $I_{1}=I_{m}$, and thus SAL.

Now we prove a proposition that relates these results to RFP, as defined in Definition 4.1.

Proposition C.9. If $\mathcal{K}$ fulfills both $S A L$ and $Z C L$, then there exist two tpCPM, $\mathcal{T}$ and $\mathcal{S}$ so that

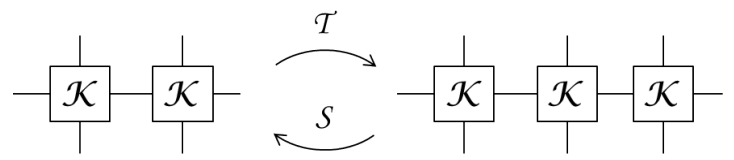

Proof. Using Corollary C.6 we can use the fact that the tensor has the form (C.5), with (C.6), (C.7), and (C.8). We will ignore the isometry $U$ from now on, as it does not add anything to the problem. Given the direct sum structure in (C.5), in order to be more concise, for each subspace labeled by $k$ we will separate the left and right spaces and call them subspins. For instance, if we have three spins, 1, 2, and 3, we will talk about the first left subspin, 11, or the second right subspin, 2r. We define the map $\mathcal{T}$ as the composition of three tpCPM, $\mathcal{T}=\mathcal{T}_{2} \mathcal{T}_{1} \mathcal{T}_{0}$. The first one, $\mathcal{T}_{0}$, transforms two spins into a single one, whereas $\mathcal{T}_{1}$ transforms one into three. In each orthogonal subspace, $k$, the first performs a trace in subspins $1 \mathrm{r}$ and $2 \mathrm{l}$, and it is thus explicitly a tpCPM. The second one, $\mathcal{T}_{1}$, introduces two operators $\eta$. More explicitly,

$$
\mathcal{T}_{1}(X)=\sum_{k, h} \mathcal{T}_{k, h}\left[\left(Q_{k}^{l} \otimes Q_{h}^{r}\right) X\left(Q_{k}^{l} \otimes Q_{h}^{r}\right)\right]
$$

Here the $Q_{k}^{l, r}$ are projectors on the left and right subspaces corresponding to the index $k$, and

$$
\mathcal{T}_{k, h}(X)=\frac{1}{a_{k} b_{h}} X \otimes\left[\oplus_{l}\left(\eta_{k, l} \otimes \eta_{l, h}\right)\right] .
$$

This is also a tpCPM, the trace-preserving property being a consequence of

$$
\frac{1}{a_{k} b_{h}} \sum_{l} \operatorname{tr}\left(\eta_{k, l}\right) \operatorname{tr}\left(\eta_{l, h}\right)=1
$$

where we have used that $\operatorname{tr}\left(\eta_{k, h}\right)=a_{k} b_{h}$ and (78). Finally, $\mathcal{T}_{2}$ just shifts in each subspace labeled by the different $k$ 's, some of the subspins. More explicitly,

$$
\mathcal{T}_{2}\left(X_{k_{1}}^{1 l} \otimes X_{h_{1}}^{1 r} X_{k_{2}}^{2 l} \otimes X_{h_{2}}^{2 r} X_{k_{3}}^{3 l} \otimes X_{h_{3}}^{3 r}\right)=X_{k_{1}}^{1 l} \otimes X_{k_{2}}^{1 r} \otimes X_{h_{2}}^{2 l} X_{k_{3}}^{2 r} \otimes X_{h_{3}}^{3 l} \otimes X_{h_{1}}^{3 r} .
$$


In summary, we have

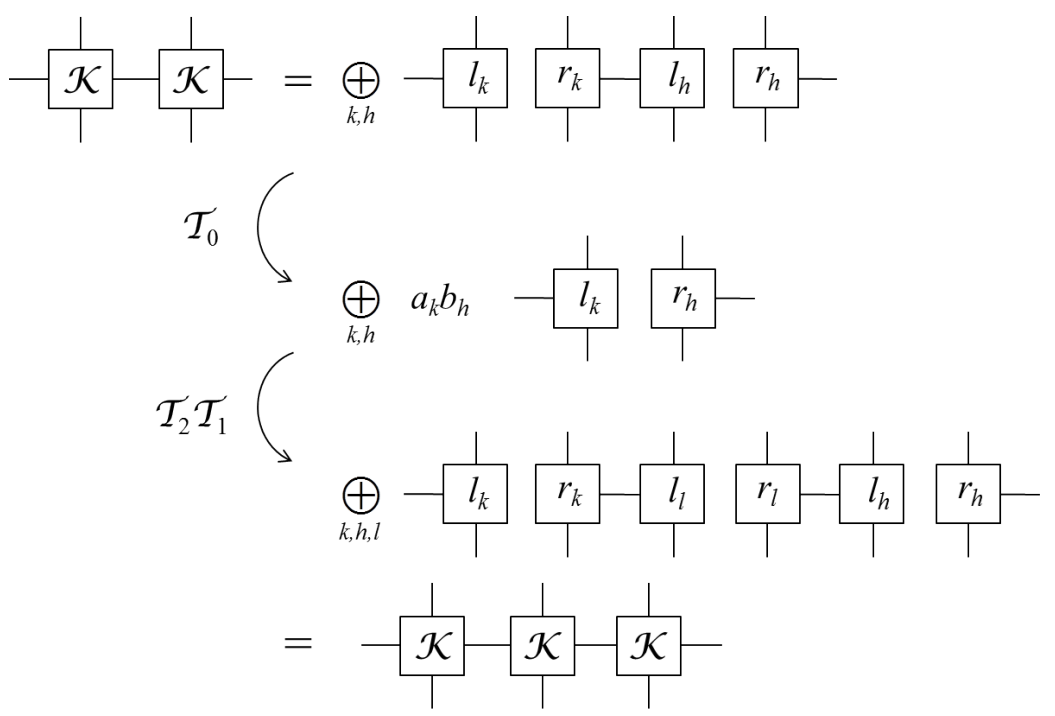

In a similar way, we build $\mathcal{S}$ out of three tpCPM, $\mathcal{S}=\mathcal{S}_{2} \mathcal{S}_{1} \mathcal{S}_{0}$. The first one, $\mathcal{S}_{0}$ in each subspace just takes the trace of the subspins $1 r, 2 l, 2 r$ and $2 l$. The second one,

$$
\mathcal{S}_{1}(X)=\sum_{k, h} \mathcal{S}_{k, h}\left[\left(Q_{k}^{l} \otimes Q_{h}^{r}\right) X\left(Q_{k}^{l} \otimes Q_{h}^{r}\right)\right]
$$

where

$$
\mathcal{S}_{k, h}(X)=\frac{1}{a_{k} b_{h}} X \otimes \eta_{k, h},
$$

so that, again, $\mathcal{S}_{1}$ is a tpCPM. The last one $\mathcal{S}_{2}$ performs a shift similar to $\mathcal{T}_{2}$,

$$
\mathcal{S}_{2}\left(X_{k_{1}}^{1 l} \otimes X_{h_{1}}^{1 r} X_{k_{2}}^{2 l} \otimes X_{h_{2}}^{2 r}\right)=X_{k_{1}}^{1 l} \otimes X_{k_{2}}^{1 r} \otimes X_{h_{2}}^{2 l} X_{h_{1}}^{2 r}
$$


and so it is tpCPM. We have

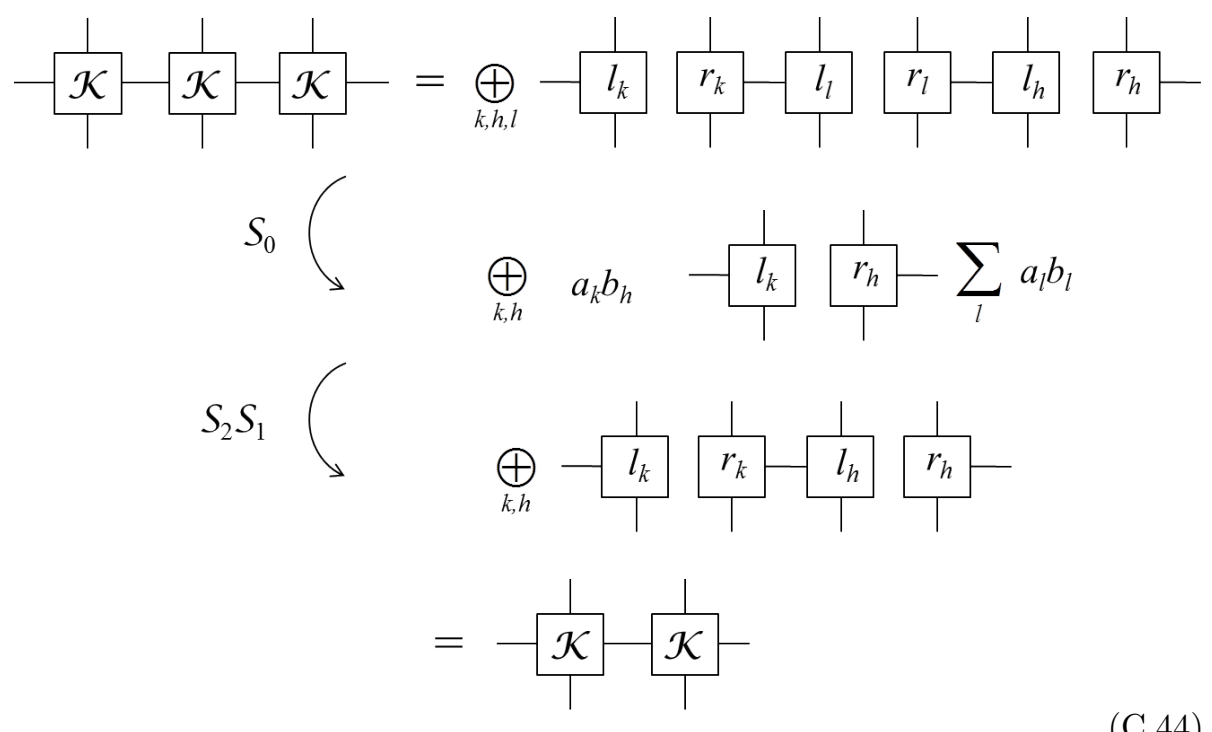

Appendix C.2.2. Case II: $K$ simple

Now we move to the more general case. The idea is to show that this case can be reduced to the injective one. Thus, from now on we will consider a tensor $K$, generating MPDO, and that is both simple and in biCF. We will use calligraphic letters, $\mathcal{K}$, to denote elements of a BNT. In case there are several elements, $\mathcal{K}_{j}$, we will use indistinguishably

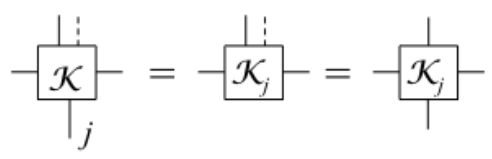

The strategy to prove Theorem 4.9 in general will be to first show that the elements in a BNT of $K$ fulfill

$$
\frac{-\mathcal{K}_{y}}{-\mathcal{K}_{x}}=0 \quad \text { if } x \neq y,
$$

and then that each of them generates MPDO and fulfills SAL and ZCL, so that we can use the results proven in the previous section. In particular, we will relate those properties to GSNNCH (see Definition 4.8):

$$
\sigma^{(N)} \propto \oplus_{x} n_{x} e^{-\sum_{j=1}^{N} \tau_{j}\left(h^{(x)}\right)} .
$$


where $n_{x}$ are natural numbers and the $h^{(x)}$ acts on the first two spins, $\tau_{j}$ translates the spins by an amount $j$, and $\left[h^{(x)}, \tau_{1}\left(h^{(x)}\right)\right]=0$.

Let us start out by showing that ZCL implies that the $\mu_{j, q}$ do not depend on $q$.

Lemma C.10. A simple tensor, $K$, generating a MPDO and fulfilling $Z C L$, must have for all $q, \mu_{j, q}=\mu_{j}$ in its CF (23).

Proof. We just have to apply the Definition 4.2 and use (23), so that

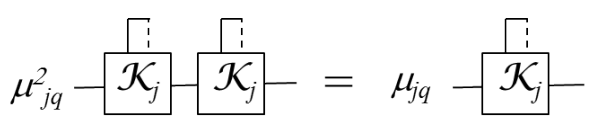

since this cannot be zero, we conclude the proof.

According to this lemma, we can include the $\mu_{j}$ in the definition of the BNT, $\mathcal{K}_{j}$, and explicitly represent this tensor in $\mathrm{CF}$ as follows [compare (23)]:

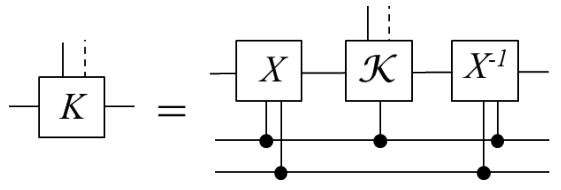

Since $K$ is in biCF (2.8), there exists another tensor, $K^{-1}$, such that

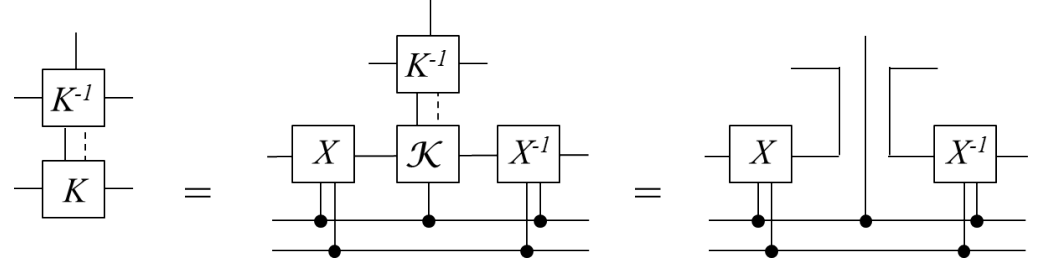

Note that this implies that

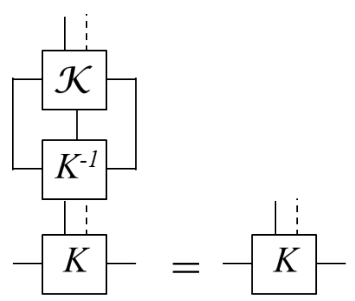

so that we recover the original tensor after applying $K^{-1}$.

We will need first some intermediate lemmas:

Lemma C.11. If $K$ fulfills $S A L$, there exist orthogonal projectors, $P_{j}$ such that

$$
\sum_{i} P_{i}=\mathbb{1}
$$


and

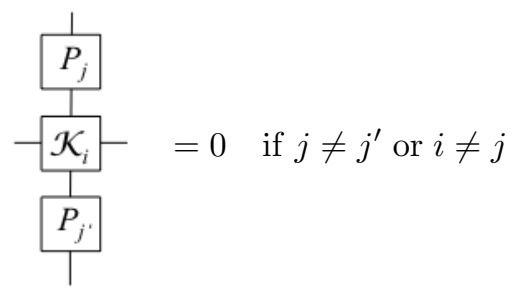

Proof. As $K$ fulfills SAL, we can use Lemma C.2, and thus $\sigma_{3}^{(N)}$ must have the form (C.3). Thus, there exist some projectors, $Q_{k}$ (see Definition C.3) that project onto each of the subspaces labeled by $k$, fulfilling

$$
\sum_{k} Q_{k}=\mathbb{1}
$$

We will now show that

$$
\begin{aligned}
& \frac{1}{Q_{k}} \\
& \frac{1}{K}-=0 \text { if } k \neq k^{\prime} \\
& Q_{k}
\end{aligned}
$$

and that for each $k$ there exists a unique $j$, such that

$$
\begin{aligned}
& \frac{1}{Q_{k}} \\
& -\mathcal{K}_{j}-\neq 0 \\
& Q_{k}
\end{aligned}
$$

We then define $S_{j}$ as the set of $k$ 's such that (C.56) holds. As a consequence that for each $k$ there exists a unique $j$ fulfilling (C.56), these sets are disjoint, and their union is the whole set of $k$ 's. Thus, defining

$$
P_{j}=\sum_{k \in S_{j}} Q_{k}
$$

we will have that they are orthogonal projectors fulfilling (C.52). Furthermore, (C.53) will follow immediately. Thus, from now on we just have to prove (C.55) and (C.56).

Using the fact that $K$ is simple, for each $j$ we can always find values of the indices, $\alpha_{j}, \beta_{j}$, such that

$$
\tilde{m}_{j}=\alpha_{j}-\Gamma_{\mathcal{K}_{j}}-\ldots-\Gamma_{i}
$$


We can also find some $N$ such that $q_{j}=\sum_{q} \mu_{j q}^{N} \neq 0$. We will consider that $N$ from now on, and denote by $m_{j}=q_{j} \tilde{m}_{j} \neq 0$. Following a similar path as in Lemma C.4, we define

$$
X_{j, \alpha, \beta}=\alpha-\frac{\mid j}{K_{T !}^{-1}-\beta}
$$

and apply them to the first and third spin of $\sigma_{3}^{(N)}$, together with the projectors $Q_{k}$ and $Q_{k^{\prime}}$ to the second one, obtaining

$$
\delta_{k, k^{\prime}} A_{j, \alpha}^{(k)} \otimes B_{j^{\prime}, \beta}^{(k)}=Q_{k} \operatorname{tr}_{1,2}\left[\left(X_{j, \beta_{j}, \alpha} \otimes \mathbb{1}_{2} \otimes X_{j^{\prime}, \beta, \alpha_{j^{\prime}}}\right) \sigma_{3}^{(N)}\right] Q_{k^{\prime}}
$$

where $A_{j, \alpha}^{(k)}=\operatorname{tr}_{1}\left(X_{j, \beta_{j}, \alpha} \rho_{A b_{1}}^{(k)}\right)$ and similarly with $B_{j^{\prime}, \beta}^{(k)}$. Taking (C.2) (with $K$ instead of $\mathcal{K}$ ) for $\sigma_{3}^{(N)}$, and using (C.50) in the computation of the rhs of (C.60) we get

$$
\delta_{k, k^{\prime}} A_{j, \alpha}^{(k)} \otimes B_{j^{\prime}, \beta}^{(k)}=\delta_{j, j^{\prime}} m_{j} \quad \alpha-\frac{\mathcal{K}_{j}-\beta}{Q_{k}}
$$

This is the main equation from which we can derive all the desired results. First, taking $j=j^{\prime}$, if $k \neq k^{\prime}$ for all $\alpha, \beta$, the lhs vanishes, so that we must have (C.55). Let us denote by

$$
O_{j}^{(k)}=-\frac{1}{Q_{k}}
$$

Now, for all $k=k^{\prime}$ there must be some $j=j^{\prime}, \alpha$ and $\beta$ such that the rhs is non-zero, since otherwise the whole $Q_{k} \sigma_{3}^{(N)} Q_{k}$ would vanish, which wlog can be assumed to be different from zero. Thus, for each $k$ there exist at least one $j_{k}$ such that $O_{j_{k}}^{(k)}$ does not vanish. Let us now show that there cannot be another $j \neq j_{k}$ with the same property. Since $O_{j_{k}}^{(k)}$ does not vanish, using (C.61) we conclude that there is some $\alpha=\alpha_{k}$ such that $A_{j_{k}, \alpha_{k}}^{(k)} \neq 0$. Using again (C.61) we conclude that for any $j \neq j_{k}$ and $\beta, B_{j, \beta}^{(k)}=0$. Thus, $A_{j, \alpha}^{(k)} \otimes B_{j, \beta}^{(k)}=0$ for all $\alpha, \beta$, and from (C.61) we obtain that $O_{j}^{(k)}=0$ as well, as we claimed, and which concludes the proof. 
Proposition C.12. If $K$ fulfills $S A L$ and $Z C L$, then the BNT's fulfill (C.46), and for each of them there exists an isometry, $U$, tensors $r_{k}$ and $l_{k}$, vectors $\Phi$ and $\Psi$, and real numbers $a_{k}, b_{k}$ such that (C.5), with (C.6), (C.7), and (C.8).

Proof. As shown in the previous lemma, there exist orthogonal projectors $P_{j}$ fulfilling (C.52) and (C.53). From those properties, one can easily show that

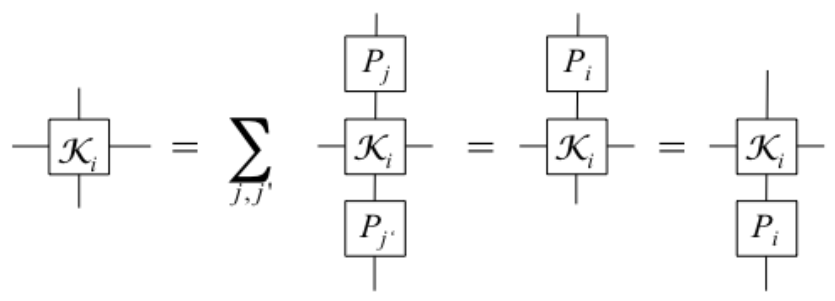

which immediately implies (C.46). Next, we will show that each BNT element $\mathcal{K}_{j}$ generates MPDO, and fulfills SAL and ZCL. Then, applying Corollary C.6, we will conclude the proof.

The fact that each $\mathcal{K}_{j}$ generates a MPDO can be shown as follows. Let us define

$$
\sigma_{j}^{(N)}(K)=P_{j}^{\otimes N} \sigma^{(N)}(K) P_{j}^{\otimes N}=n_{j} \sigma^{(N)}\left(\mathcal{K}_{j}\right)
$$

where we have used (C.63) and Lemma C.10, and where $n_{j}$ is the number of blocks with index $j$. From this identity, we have that $\sigma^{(N)}\left(\mathcal{K}_{j}\right) \geq 0$.

In order to show that each BNT fulfills SAL, let us divide a chain of $N$ spins into four regions, A,B,C, and D, containing $m-1,1, N-m-1$, and 1 consecutive spins, respectively. We can write

$$
\sigma^{(N)}(K)=\oplus_{j} P_{j}^{(B)} \sigma^{(N)}(K) P_{j}^{(B)}=\oplus_{j} \sigma_{j}^{(N)}(K)
$$

where the projectors are applied to the spin in region B only, and we have used (C.63). Let us denote by $p_{j}=\operatorname{tr}\left[\sigma_{j}^{(N)}(K)\right] \geq 0$, by $S_{X}^{j}$ the entropy of $\sigma_{j}^{(N)}(K) / p_{j}$ in region $X$, and by $H_{p}=-\sum_{j} p_{j} \log p_{j}$. We will also denote by $S_{X}$ the entropy of $\sigma^{(N)}(K)$ in region $X$. Given that the different $\sigma_{j}^{(N)}(K)$ are supported on different subspaces in region $B$, we can write

$$
S_{X}=H_{p}+\sum_{j} p_{j} S_{X}^{j}
$$

Now, using the fact that $K$ fulfills SAL, and thus $I_{1}=I_{m}$ for $m<\lfloor N / 2\rfloor$, this implies $S_{A B C}+S_{B}=S_{A B}+S_{B C}$. Using (C.66) we obtain

$$
\sum_{j} p_{j}\left(S_{A B C}^{j}+S_{B}^{j}\right)=\sum_{j} p_{j}\left(S_{A B}^{j}+S_{B C}^{j}\right) .
$$

Together with the strong subadditivity inequality for each of the $\sigma_{j}^{(N)}(K)$, i.e.

$$
S_{A B C}^{j}+S_{B}^{j} \leq S_{A B}^{j}+S_{B C}^{j}
$$


this implies that for each $j$, the (C.68) must be fulfilled as an equality, if one of them would be strictly smaller, then it would be impossible to have (C.67). Substracting $S_{N}^{j}$ on each side of the equality we obtain that the mutual information is independent of $m$, and thus that each $\sigma_{j}^{(N)}(K) / p_{j}$ saturates the area law.

Using the relation between $\sigma_{j}^{(N)}(K)$ and $\sigma^{(N)}\left(\mathcal{K}_{j}\right)$ given in (C.64), we conclude that each $\mathcal{K}_{j}$ fulfills SAL. Furthermore, since $K$ has ZCL then using the CF (C.49) it is obvious that each $\mathcal{K}_{j}$ must also have ZCL.

Next, we prove the following

Proposition C.13. If the BNT's of $K$ fulfill (C.46), and (C.5), with (C.6), (C.7), and (C.8), then the MPDO generated by $K$ have the form (C.47).

Proof. Given (C.46) and Lemma C.10, we can write

$$
\sigma^{(N)}(K)=\oplus_{j} n_{j} \sigma\left(\mathcal{K}_{j}\right)
$$

where $n_{j}$ is an integer. The rest follows directly from Proposition C.7 since, according to the previous proposition, each $\mathcal{K}_{j}$ fulfills SAL and ZCL.

Now we show

Proposition C.14. If $K$ generates MPDO of the form (C.47) then it fulfills $S A L$.

Proof. Using Proposition C.8 to each of the terms in the direct sum we have that each $\mathcal{K}_{j}$ fulfills SAL. Since the direct sum is local, this also applies to the whole $K$.

Proposition C.15. If $K$ fulfills $S A L$ and $Z C L$ then there exist two tpCPM $\mathcal{T}$ and $\mathcal{S}$ fulfilling (C.35).

Proof. The proof follows in the same way as that of Proposition C.9 by first projecting into each local subspace labeled by $j$.

Now we are at the position of proving Theorem 4.9:

Proof. $i \Rightarrow i i$ by Proposition C.1. $\quad$ ii $\Rightarrow i i i$ by Proposition C.12. $i i i \Rightarrow i v$ by Proposition C.13. $i v \Rightarrow i i$ by Proposition C.14. $\quad$ iii $\Rightarrow v$ by Proposition C.15 where the tpCPM are $\mathcal{T}^{2}$ and $\mathcal{S}^{2}$.

Appendix C.3. Proof of Proposition 4.13.

In order to prove it, we will need the following Lemma about MPV.

Lemma C.16. Let $A$ be a tensor in $C F$ generating a family of $M P V$, with local Hilbert space $\mathcal{H}_{d}$. Let $Y, Z$ be operators on $\mathcal{H}_{d}$ If $Y_{1} V^{(N)}(A)=Z_{1} V^{(N)}(A)$ for 
all $N$ (where $Y_{1}, Z_{1}$ means that the operators are located in the first spin) graphically

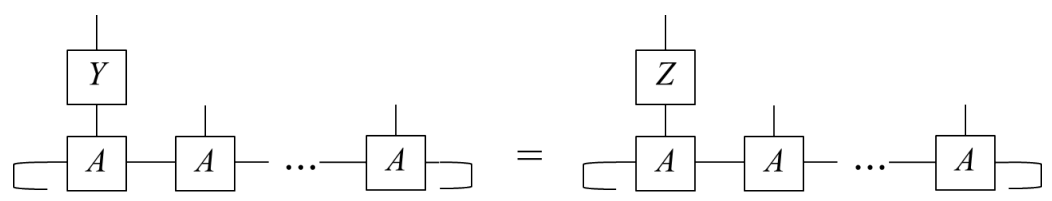

then

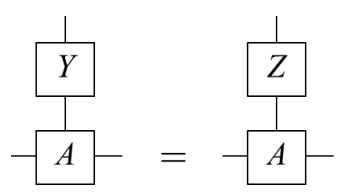

Proof. Let us consider the CF decomposition of $A$ given in (20a). The hypothesis implies that

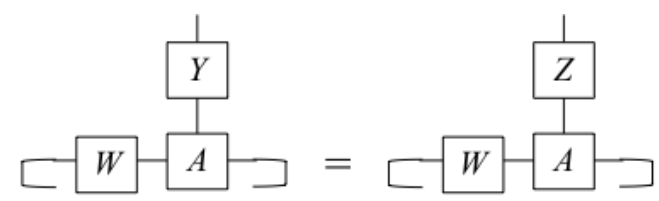

for all $W \in \operatorname{span}\left\{\oplus_{j, q} \mu_{j, q} X_{j, q} A_{j} X_{j, q}^{-1}\right\}^{N}$. That is, calling

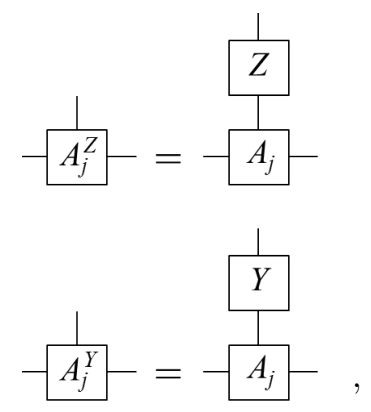

Proposition 2.9 ensures that

$$
\left(\sum_{q} \mu_{j, q}^{N}\right) \operatorname{tr}\left[W_{j} A_{j}^{Y}\right]=\left(\sum_{q} \mu_{j, q}^{N}\right) \operatorname{tr}\left[W_{j} A_{j}^{Z}\right]
$$

for all $j$, all blocks $W_{j}$ of $W$, and all $N$. By choosing $N$ so that $\sum_{q} \mu_{j, q}^{N} \neq 0$, which exists by Lemma A.5, we get that $A_{j}^{Y}=A_{j}^{Z}$ for all $j$ and from there the desired result.

We can now prove Proposition 4.13.. Let us first restate it. 
Proposition. A tensor $\tilde{M}$ in $C F$, generating MPDO, is also in $C F$ vertically. Moreover, there exists an isometry $U$ such that

$$
U \tilde{M} U^{\dagger}=\bigoplus_{\alpha} \mu_{\alpha} \otimes M_{\alpha}
$$

where $\mu_{\alpha}$ are diagonal and positive matrices, and $\left\{M_{\alpha}\right\}_{\alpha}$ is a BNT.

Proof. Let us first show that for all projector $P$ so that $P \tilde{M}=P \tilde{M} P$, we have that $P^{\perp} \tilde{M} P=0$.

Let us denote by $H^{(N)}=H^{(N)}(\tilde{M})$ the MPDO generated by $\tilde{M}$ in the horizontal direction. That is

$$
H^{(N)}(\tilde{M})=\square \sqrt{\frac{1}{M}} \ldots-\frac{1}{\tilde{M}} \square
$$

Imagine there exists a projector $P$ so that $P \tilde{M}=P \tilde{M} P$. Then

$$
P H^{(N)}={ }^{(N)} P=\left(P H^{(N)} P\right)^{\dagger}=H^{(N)} P
$$

By assumption, $\tilde{M}$ is in $\mathrm{CF}$ in the horizontal direction. Then, Lemma C.16 gives $P^{\perp} \tilde{M} P=0$ as desired.

The next step is to show that there are no p-periodic vectors. If this were the case, there would exist an orthogonal projector $Q$ so that $Q H^{(N)} \neq H^{(N)} Q$ for all $N$ but

$$
Q\left[H^{(N)}\right]^{p}=\left[H^{(N)}\right]^{p} Q \text { for all } N .
$$

But since $H^{(N)}$ is semidefinite positive, (C.73) implies that $Q H^{(N)}=H^{(N)} Q$ for all $N$, which is the desired contradiction.

It only remains to show that there exists an isometry $U$ so that

$$
U \tilde{M} U^{\dagger}=\bigoplus_{\alpha} \mu_{\alpha} \otimes M_{\alpha}
$$

where the $\mu_{\alpha}$ are positive diagonal matrices and the $M_{\alpha}$ a BNT for $\tilde{M}$ in the vertical direction.

Since $\tilde{M}$ is in CF in the vertical direction, it can be written as $\oplus_{\alpha, k} \mu_{\alpha, k} X_{\alpha, k} M_{\alpha} X_{\alpha, k}^{-1}$. Let us denote by $P_{\alpha, k}$ the projector selecting the sector $\alpha, k$ in such direct sum. Then, since $\tilde{M}$ was also in CF in the horizontal direction, there exists an $N$ so 
that $0 \neq P_{\alpha, k} H^{(N)} P_{\alpha, k} \geq 0$. Its trace is then $\mu_{\alpha, k} d_{\alpha}>0$, where

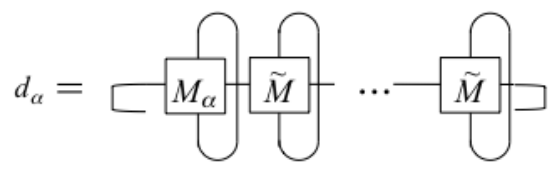

Since wlog $\mu_{\alpha, 1}>0$ for all $\alpha$, we can conclude that $d_{\alpha}>0$ and hence $\mu_{\alpha, k}>0$. We now finish if we show that

$$
X_{\alpha, k}^{\dagger} X_{\alpha, k}=\omega_{\alpha, k} X_{\alpha, 1}^{\dagger} X_{\alpha, 1} \text { for all } k .
$$

Since we can always choose wlog $X_{\alpha, 1}=\mathbb{1}$ for all $\alpha$, this would imply that

$$
\frac{1}{\sqrt{\omega_{\alpha, k}}} X_{\alpha, k}=U_{\alpha, k}
$$

Since the $X_{\alpha, k}$ are defined up to a constant, we are done.

Let us prove then (C.75). Using that $P_{\alpha, k} H^{(N)} P_{\alpha, k}$ is self-adjoint and that $\mu_{\alpha, k}>0$ we arrive at

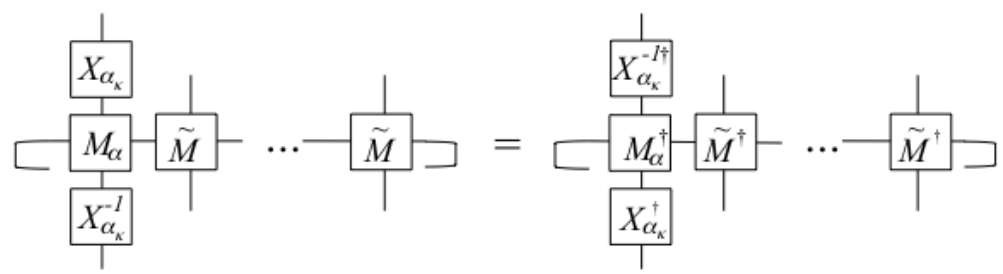

Using that $\tilde{M}$ is in $\mathrm{CF}$ in the horizontal direction together with Lemma C.16 we get :

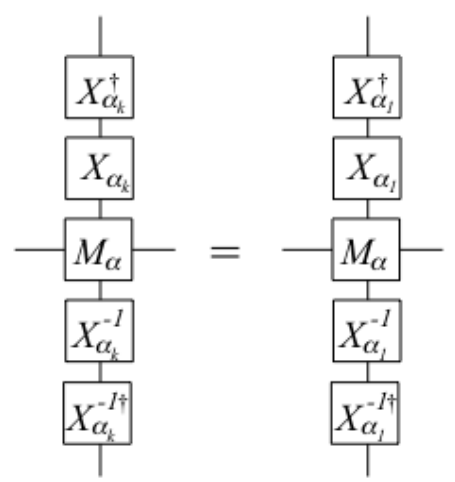

But since $M_{\alpha}$ is a NT in the vertical direction, we obtain (C.75) for a (necessarily positive) constant $\omega_{\alpha, k}$. 
Appendix C.4. Proof of Theorem 4.14

Let us restate for convenience here Theorem 4.14

Theorem. Given a tensor $M$ in $C F$ that generates $M P D O$, the following statements are equivalent:

(i) $M$ is a RFP.

(ii) There exists a set of diagonal matrices, $\chi_{\alpha, \beta, \gamma}$, with positive elements, such that for each $L$ the operators $O_{L}\left(M_{\alpha}\right)$ linearly span an algebra with structure coefficients $c_{\alpha, \beta, \gamma}^{(L)}=\operatorname{tr}\left(\chi_{\alpha, \beta, \gamma}^{L}\right)$ :

$$
O_{L}\left(M_{\alpha}\right) O_{L}\left(M_{\beta}\right)=\sum_{\gamma} c_{\alpha, \beta, \gamma}^{(L)} O_{L}\left(M_{\gamma}\right)
$$

and

$$
m_{\gamma}=\sum_{\alpha, \beta} c_{\alpha, \beta, \gamma}^{(1)} m_{\alpha} m_{\beta}
$$

where $m_{\alpha}=\operatorname{tr}\left(\mu_{\alpha}\right)$. That is, the vector $\left(m_{\alpha}\right)_{\alpha}$ is an idempotent for the "multiplication" induced by $c^{(1)}$.

(iii) There exist isometries, $U_{\alpha, \beta}$, such that

$$
U_{\alpha, \beta} M_{\alpha} M_{\beta} U_{\alpha, \beta}^{\dagger}=\oplus_{\gamma} \chi_{\alpha, \beta, \gamma} \otimes M_{\gamma},
$$

where $\chi_{\alpha, \beta, \gamma}$ are the same as in the previous statement, and (C.79) is fulfilled.

Proof. $(i) \Rightarrow(i i)$ and (iii). By Proposition 4.13, both

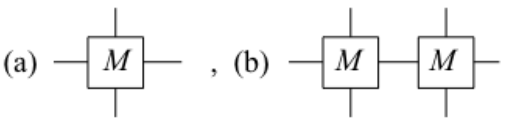

are in $\mathrm{CF}$ in vertical direction. So, up to isometries that can be absorbed in the $T$ and $S$ appearing in the RFP property, they can be written as $\oplus_{\alpha} \mu_{\alpha} \otimes M_{\alpha}$ and $\oplus_{\beta} \nu_{\beta} \otimes A_{\beta}$, respectively, where $\left\{M_{\alpha}\right\}_{\alpha}$ and $\left\{A_{\beta}\right\}_{\beta}$ are BNT with dimensions $d_{\alpha}^{1}$ and $d_{\beta}^{2}$, respectively, and $\mu_{\alpha}, \nu_{\beta}$ are diagonal positive matrices with $m_{\alpha}=$ $\operatorname{tr}\left[\mu_{\alpha}\right]$ and $n_{\beta}=\operatorname{tr}\left[\nu_{\beta}\right]$. Let us define the $C^{*}$-algebras $\mathcal{A}_{1}=\oplus_{\alpha} \mathcal{M}_{d_{\alpha}^{1} \times d_{\alpha}^{1}}$ and $\mathcal{A}_{2}=\oplus_{\beta} \mathcal{M}_{d_{\beta}^{2} \times d_{\beta}^{2}}$ and the operators

$$
\begin{aligned}
& R_{1}: \mathcal{A}_{1} \longrightarrow \oplus_{\alpha} \operatorname{span}\left\{\mu_{\alpha}\right\} \otimes \mathcal{M}_{d_{\alpha}^{1} \times d_{\alpha}^{1}}, \\
& R_{2}: \mathcal{A}_{2} \longrightarrow \oplus_{\beta} \operatorname{span}\left\{\nu_{\beta}\right\} \otimes \mathcal{M}_{d_{\beta}^{2} \times d_{\beta}^{2}}, \\
& \tilde{R}_{1}: \oplus_{\alpha} \operatorname{span}\left\{\mu_{\alpha}\right\} \otimes \mathcal{M}_{d_{\alpha}^{1} \times d_{\alpha}^{1}} \longrightarrow \mathcal{A}_{1} \\
& \tilde{R}_{2}: \oplus_{\beta} \operatorname{span}\left\{\nu_{\beta}\right\} \otimes \mathcal{M}_{d_{\beta}^{2} \times d_{\beta}^{2}} \longrightarrow \mathcal{A}_{2}
\end{aligned}
$$


by

$$
\begin{aligned}
R_{1}\left(\oplus_{\alpha} X_{\alpha}\right) & =\oplus_{\alpha} \frac{\mu_{\alpha}}{m_{\alpha}} \otimes X_{\alpha} \\
R_{2}\left(\oplus_{\beta} Y_{\beta}\right) & =\oplus_{\beta} \frac{\nu_{\beta}}{n_{\beta}} \otimes Y_{\beta} \\
\tilde{R}_{1}\left(\oplus_{\alpha} \lambda_{\alpha} \mu_{\alpha} \otimes X_{\alpha}\right) & =\oplus_{\alpha} \lambda_{\alpha} m_{\alpha} X_{\alpha} \\
\tilde{R}_{2}\left(\oplus_{\beta} \delta_{\beta} \nu_{\beta} \otimes Y_{\beta}\right) & =\oplus_{\beta} \delta_{\beta} n_{\beta} Y_{\beta}
\end{aligned}
$$

Let us call $\tilde{T}=\tilde{R}_{2} T R_{1}: \mathcal{A}_{1} \rightarrow \mathcal{A}_{2}$ and $\tilde{S}=\tilde{R}_{1} S R_{2}: \mathcal{A}_{2} \rightarrow \mathcal{A}_{1}$, where $T, S$ are the tpCPM in the definition of RFP. Let us show that $\tilde{S} \tilde{T}$ is the identity in $\mathcal{A}_{1}$ and $\tilde{T} \tilde{S}$ the identity in $\mathcal{A}_{2}$. For that we note that $\tilde{T}\left(\oplus_{\alpha} m_{\alpha} M_{\alpha}(X)\right)=$ $\oplus_{\beta} n_{\beta} A_{\beta}(X)$ and $\tilde{S}\left(\oplus_{\beta} n_{\beta} A_{\beta}(X)\right)=\oplus_{\alpha} m_{\alpha} M_{\alpha}(X)$, where

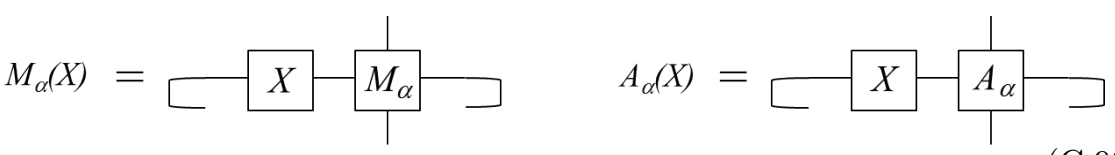

Hence $\oplus_{\alpha} m_{\alpha} M_{\alpha}(X)$ is contained in the set of fixed points $\mathcal{F}$ of $\tilde{S} \tilde{T}$. By [34, Theorem 6.14], $\mathcal{F}$ has the form

$$
\mathcal{F}=U\left[0_{r} \oplus_{k} \rho_{k} \otimes \mathcal{M}_{h_{k} \times h_{k}}\right] U^{\dagger}
$$

for a unitary $U$. Given a vector subspace $V$ of $\mathcal{M}_{D \times D}$ and $L \in \mathbb{N}$, we call

$$
C_{L}(V)=\operatorname{span}\left(V^{L}\right)=\operatorname{span}\left\{v_{1} v_{2} \cdots v_{L}: v_{i} \in V\right\} .
$$

Since $V=\left\{\oplus_{\alpha} m_{\alpha} M_{\alpha}(X): X\right\} \subset \mathcal{F}$, we have that $C_{L}(V) \subset C_{L}(\mathcal{F})$ for all $L$. But Proposition 2.9 guarantees that for some $L, C_{L}(V)=\mathcal{A}_{1}$. Moreover

$$
C_{L}(\mathcal{F})=U\left[0_{r} \oplus_{k} \rho_{k}^{L} \otimes \mathcal{M}_{h_{k} \times h_{k}}\right] U^{\dagger} .
$$

This clearly implies that $\mathcal{F}=\mathcal{A}_{1}$ and hence $\tilde{S} \tilde{T}$ is the identity in $\mathcal{A}_{1}$.

A similar argument proves that $\tilde{T} \tilde{S}$ the identity in $\mathcal{A}_{2}$.

Reasoning as in [38, Theorem 8] we get that (after relabeling of blocks that can be absorbed in the definition of $T$ and $S), \mathcal{A}_{1}=\mathcal{A}_{2}$ and $\tilde{T}\left(\oplus X_{\alpha}\right)=$ $\oplus_{\alpha} U_{\alpha} X_{\alpha} U_{\alpha}^{\dagger}, \tilde{S}\left(\oplus Y_{\alpha}\right)=\oplus_{\alpha} U_{\alpha}^{\dagger} Y_{\alpha} U_{\alpha}$ for some unitaries $U_{\alpha}$.

Now

$$
\begin{gathered}
\oplus_{\alpha} \nu_{\alpha} \otimes A_{\alpha}(X)=T\left(\oplus_{\alpha} \mu_{\alpha} \otimes M_{\alpha}(X)\right)= \\
=R_{2} \tilde{T} \tilde{R}_{1}\left(\oplus_{\alpha} \mu_{\alpha} \otimes M_{\alpha}(X)\right)=\oplus_{\alpha} \frac{m_{\alpha}}{n_{\alpha}} \nu_{\alpha} \otimes U_{\alpha} M_{\alpha}(X) U_{\alpha}^{\dagger}
\end{gathered}
$$

from where we conclude (remember we did a relabelling of blocks)

$$
A_{\alpha}=\frac{m_{\alpha}}{n_{\alpha}} U_{\alpha} M_{\alpha} U_{\alpha}^{\dagger}, \forall \alpha
$$


Then, if we denote (C.81.(b)) by $B$, we get that $O_{L}(B)$ can be written simultaneously as

$$
O_{L}(B)=\sum_{\gamma} \frac{m_{\gamma}^{L}}{n_{\gamma}} \operatorname{tr}\left[\nu_{\gamma}^{L}\right] O_{L}\left(M_{\gamma}\right)
$$

and

$$
O_{L}(B)=\sum_{\alpha, \beta} \operatorname{tr}\left[\mu_{\alpha}^{L}\right] \operatorname{tr}\left[\mu_{\beta}^{L}\right] O_{L}\left(M_{\alpha}\right) O_{L}\left(M_{\beta}\right)
$$

From here, it is easy to see that a BNT for

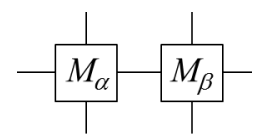

must contain neccessarily only elements of the form $e^{i \theta_{\alpha, \beta, \gamma}} Z_{\alpha, \beta, \gamma} M_{\gamma} Z_{\alpha, \beta, \gamma}^{-1}$ so that

$$
(\mathrm{C} .99)=\oplus_{\gamma} \chi_{\alpha, \beta, \gamma} \otimes Z_{\alpha, \beta, \gamma} M_{\gamma} Z_{\alpha, \beta, \gamma}^{-1}
$$

for a diagonal matrix $\chi_{\alpha, \beta, \gamma}$. This gives (C.78). Reasoning as in Proposition 4.13 we get that $\chi_{\alpha, \beta, \gamma}$ are positive and $Z_{\alpha, \beta, \gamma}$ unitaries. It only remains to show (C.79). By plugging (C.100) into (C.98) we get

$$
\begin{gathered}
\sum_{\gamma} \frac{m_{\gamma}^{L}}{n_{\gamma}} \operatorname{tr}\left[\nu_{\gamma}^{L}\right] O_{L}\left(M_{\gamma}\right)= \\
=O_{L}(B)=\sum_{\alpha, \beta, \gamma} \operatorname{tr}\left[\left(\mu_{\alpha} \otimes \mu_{\beta} \otimes \chi_{\alpha, \beta, \gamma}\right)^{L}\right] O_{L}\left(M_{\gamma}\right)
\end{gathered}
$$

Since the $M_{\gamma}$ form a BNT, for all $\gamma$ and for all sufficiently large $L$,

$$
\operatorname{tr}\left[\left(\frac{m_{\gamma}}{n_{\gamma}} \nu_{\gamma}\right)^{L}\right]=\sum_{\alpha, \beta} \operatorname{tr}\left[\left(\mu_{\alpha} \otimes \mu_{\beta} \otimes \chi_{\alpha, \beta, \gamma}\right)^{L}\right]
$$

By Lemma A.5, for all $\gamma$ the set $\left\{\frac{m_{\gamma}}{n_{\gamma}} \nu_{\gamma, k}\right\}_{k}$ coincides with the set $\left\{\mu_{\alpha, k_{1}} \mu_{\beta, k_{2}} \chi_{\alpha, \beta, \gamma, k_{3}}\right\}_{\alpha, \beta, k_{1}, k_{2}, k_{3}}$ (note that all coefficients are positive). In particular, summing over all indices we get (C.79).

Clearly $(i i i) \Rightarrow(i i)$ so it only remains to show that $(i i) \Rightarrow(i)$.

As above, we consider (C.81) and write them, up to conjugation by unitaries, as $\oplus_{\alpha} \mu_{\alpha} \otimes M_{\alpha}$ and $\oplus_{\beta} \nu_{\beta} \otimes A_{\beta}$. (C.78) implies that for all $L$,

$$
\sum_{\gamma} \operatorname{tr}\left[\nu_{\gamma}^{L}\right] O_{L}\left(A_{\gamma}\right)=\sum_{\alpha, \beta, \gamma} \operatorname{tr}\left[\left(\mu_{\alpha} \otimes \mu_{\beta} \otimes \chi_{\alpha, \beta, \gamma}\right)^{L}\right] O_{L}\left(M_{\gamma}\right)
$$

Since both $A_{\gamma}$ and $M_{\gamma}$ are BNT, this implies that, up to relabelling,

$$
A_{\gamma}=e^{i \theta_{\gamma}} Z_{\gamma} M_{\gamma} Z_{\gamma}^{-1}
$$


By Proposition $4.13 e^{i \theta_{\gamma}}=1$ and $Z_{\gamma}=U_{\gamma}$ is unitary. Since, for sufficiently large $L, O_{L}\left(A_{\gamma}\right)=O_{L}\left(M_{\gamma}\right)$ are linearly independent, and all coefficients $\nu_{\gamma, k}, \mu_{\alpha, k_{1}}$, $\mu_{\beta, k_{2}}, \chi_{\alpha, \beta, \gamma, k_{3}}$ are positive, Lemma A.5 implies that for all $\gamma$ the set $\left\{\nu_{\gamma, k}\right\}_{k}$ coincides with the set $\left\{\mu_{\alpha, k_{1}} \mu_{\beta, k_{2}} \chi_{\alpha, \beta, \gamma, k_{3}}\right\}_{\alpha, \beta, k_{1}, k_{2}, k_{3}}$. If we sum in all indices we get that

$$
\begin{aligned}
\operatorname{tr}\left[\nu_{\gamma}\right]=\sum_{k} \nu_{\gamma, k} & =\sum_{\alpha, \beta, k_{1}, k_{2}, k_{3}} \mu_{\alpha, k_{1}} \mu_{\beta, k_{2}} \chi_{\alpha, \beta, \gamma, k_{3}}= \\
& =\sum_{\alpha, \beta} m_{\alpha} m_{\beta} c_{\alpha, \beta, \gamma}^{(1)}=m_{\gamma}
\end{aligned}
$$

by (C.79). We can now define $T=\tilde{R}_{2} \tilde{T} R_{1}$ where

- $R_{1}: \mathcal{M}_{d \times d} \longrightarrow \oplus_{\gamma} \mathcal{M}_{d_{\gamma} \times d_{\gamma}}$ is defined by $R_{1}(X)=\sum_{\gamma} \operatorname{tr}_{\gamma, 1}\left[P_{\gamma} X P_{\gamma}\right]$, being $P_{\gamma}$ the projector on the corresponding sector of the decomposition $\oplus \mu_{\gamma} \otimes M_{\gamma}$ and $\operatorname{tr}_{\gamma, 1}$ the partial trace on the first subsystem of that sector.

- $\tilde{T}: \oplus_{\gamma} \mathcal{M}_{d_{\gamma} \times d_{\gamma}} \longrightarrow \oplus_{\gamma} \mathcal{M}_{d_{\gamma} \times d_{\gamma}}$ is just conjugation by $\oplus_{\gamma} U_{\gamma}$ and

- $\tilde{R}_{2}: \oplus_{\gamma} \mathcal{M}_{d_{\gamma} \times d_{\gamma}} \longrightarrow \mathcal{M}_{d \times d} \otimes \mathcal{M}_{d \times d}$ is given by $\tilde{R}_{2}\left(\oplus_{\gamma} X_{\gamma}\right)=\oplus_{\gamma} \frac{\nu_{\gamma}}{m_{\gamma}} \otimes X_{\gamma}$.

It is clear that $T\left(\oplus_{\gamma} \mu_{\gamma} \otimes M_{\gamma}\right)=\oplus_{\gamma} \nu_{\gamma} \otimes A_{\gamma}$ as desired in the definition of RFP.

Similarly we can define $S=\tilde{R}_{1} \tilde{S} R_{2}$ where

- $R_{2}: \mathcal{M}_{d \times d} \otimes \mathcal{M}_{d \times d} \longrightarrow \oplus_{\gamma} \mathcal{M}_{d_{\gamma} \times d_{\gamma}}$ is defined by $R_{2}(X)=\sum_{\gamma} \operatorname{tr}_{\gamma, 1}\left[Q_{\gamma} X Q_{\gamma}\right]$, being $Q_{\gamma}$ the projector on the corresponding sector of the decomposition $\oplus \nu_{\gamma} \otimes A_{\gamma}$ and $\operatorname{tr}_{\gamma, 1}$ the partial trace on the first subsystem of that sector.

- $\tilde{S}: \oplus_{\gamma} \mathcal{M}_{d_{\gamma} \times d_{\gamma}} \longrightarrow \oplus_{\gamma} \mathcal{M}_{d_{\gamma} \times d_{\gamma}}$ is just conjugation by $\oplus_{\gamma} U_{\gamma}^{\dagger}$ and

- $\tilde{R}_{1}: \oplus_{\gamma} \mathcal{M}_{d_{\gamma} \times d_{\gamma}} \longrightarrow \mathcal{M}_{d \times d}$ is defined by $\tilde{R}_{1}\left(\oplus_{\gamma} X_{\gamma}\right)=\oplus_{\gamma} \frac{\mu_{\gamma}}{m_{\gamma}} \otimes X_{\gamma}$.

It is clear that $S$ has the property $S\left(\oplus_{\gamma} \nu_{\gamma} A_{\gamma}\right)=\oplus_{\gamma} \mu_{\gamma} M_{\gamma}$ as desired in the definition of RFP. This finishes the proof of Theorem 4.14.

\section{Appendix D. Additional results}

In this Appendix we elaborate on some of the definitions and results obtained in this paper. In particular, we give alternative definitions of RFP, and exploit the relation between commuting Hamiltonians and the fact that certain correlation functions vanish. 
Appendix D.1. Alternative definitions of RFP

There are in principle other possible definitions of RFP for a tensor. The results of this paper show that the chosen ones seem to be the right ones, given their clear connection with relevant physical notions such as zero-correlation length, saturation of the area law, commuting Hamiltonians or topological properties of boundary theories. It is however worth mentioning other possibilities and their limitations.

The first possibility would be to allow in Definition 4.1 that the maps $T$ and $S$ introduce a gauge transformation in the virtual level of the MPDO.

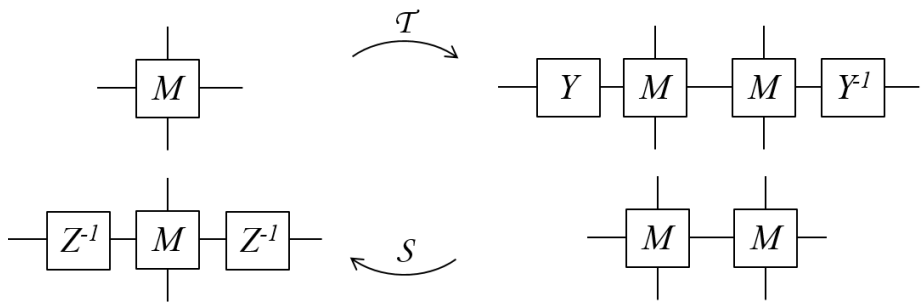

In the pure case, where $T$ and $S$ are unitary conjugations, Definition (D.1) coincides with Definition 4.1 for tensors in $\mathrm{CF}$ as the following argument shows. (D.1) implies that $\mathbb{E}^{2}$ is similar to $\mathbb{E}$ and hence both must have eigenvalues 1 and 0 exclusively. By going to CFII, one may assume wlog that $\mathbb{E}$ is contractive for the trace norm and hence a standard argument [28] shows that the eigenvalue 1 cannot have non-trivial Jordan blocks. Using that $\mathbb{E}^{k}$ is similar to $\mathbb{E}$ for all $k$ one gets that the eigenvalue 0 cannot have non-trivial Jordan blocks either. As a consequence $\mathbb{E}^{2}=\mathbb{E}$ and, by the characterization given in Theorem 3.10, the tensor is an RFP.

Unfortunately we do not know whether for mixed states a similar argument can be applied and Definition (D.1) coincides with Definition 4.1. We leave it as an interesting open question.

Another possibility could be to impose that $T$ and $S$ are given by unitary conjugations also in the mixed case. Graphically,

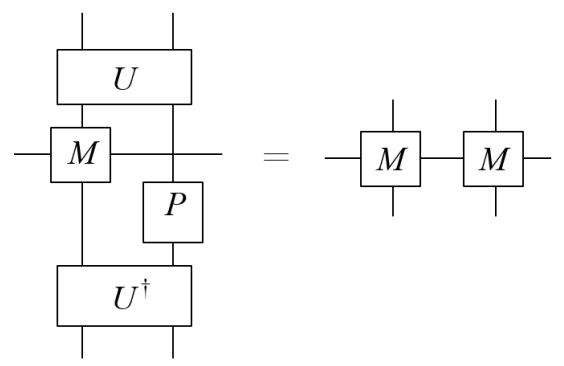

for some $P \geq 0$.

This definition is, however, too strong and many of the interesting examples arising from string-net models would not be covered. The reason is that, with (D.2), the rank of the associated MPDO of $N$ sites must grow with $N$ as $r s^{N-1}$ 
for natural numbers $r, s$. Boundary theories of many string-net models, such as the Fibonacci model, cannot have such behavior. As an illustration, let us see it for one of the elements in the BNT of the boundary theory associated to the Fibonacci model. Concretely, the one corresponding to the vacuum [23]. The tensor $A$ has bond dimension 2 and three physical qubits that we call $i j k$. For each qubit, the ket index is equal to the bra index (the tensor $A$ generates MPDO which are diagonal in the computational basis). Therefore, $A$ gets defined by the elements $A_{\alpha, \beta}^{i j k}$ and the generated MPDO are given by $\rho^{(N)}(A)=$

$\sum_{i_{1}, j_{1}, k_{1}, \ldots, i_{N}, j_{N}, k_{N}} \operatorname{tr}\left(A^{i_{1}, j_{1}, k_{1}} \cdots A^{i_{N}, j_{N}, k_{N}}\right)\left|i_{1} j_{1} k_{1} \cdots i_{N} j_{N} k_{N}\right\rangle\left\langle i_{1} j_{1} k_{1} \cdots i_{N} j_{N} k_{N}\right|$.

All we will need here is that $A_{\alpha, \beta}^{i j k}=\delta_{i, \alpha} \delta_{k, \beta} N_{i j k}$ and $N_{i j k}=0$ if and only if exactly two of the $i, j, k$ are 0 ; otherwise $N_{i j k}>0$. (This corresponds to the fusion rules of the Fibonacci model.) Let us see that

$$
\operatorname{rank}\left(\rho^{(N)}(A)\right)=\tau_{+}^{2 N}+\tau_{-}^{2 N} .
$$

where $\tau_{ \pm}=\frac{1 \pm \sqrt{5}}{2}$. It is trivial to check that $\tau_{+}^{2 N}+\tau_{-}^{2 N}$ is not of the form $r s^{N-1}$.

Denote by $x_{\alpha \beta}^{n}$ the rank of the MPDO of length $n$ generated by $A$ with left and right boundary conditions given by $\alpha, \beta \in\{0,1\}$ respectively:

$$
\sum_{i_{1}, j_{1}, k_{1}, \ldots, i_{N}, j_{N}, k_{N}}\left(\alpha\left|A^{i_{1}, j_{1}, k_{1}} \cdots A^{i_{N}, j_{N}, k_{N}}\right| \beta\right)\left|i_{1} j_{1} k_{1} \cdots i_{N} j_{N} k_{N}\right\rangle\left\langle i_{1} j_{1} k_{1} \cdots i_{N} j_{N} k_{N}\right| .
$$

Clearly, the $x^{n}$ satisfy the following recurrence relations:

$$
\begin{aligned}
& x_{00}^{n}=x_{00}^{n-1}+x_{01}^{n-1} \\
& x_{01}^{n}=x_{00}^{n-1}+2 x_{01}^{n-1} \\
& x_{10}^{n}=x_{10}^{n-1}+x_{11}^{n-1} \\
& x_{11}^{n}=x_{10}^{n-1}+2 x_{11}^{n-1} .
\end{aligned}
$$

Let us call $x^{n}$ to the vector $\left(x_{00}^{n}, x_{01}^{n}, x_{10}^{n}, x_{11}^{n}\right)$. We have $x^{1}=(1,1,1,2), x^{n}=$ $x^{n-1} A=x^{1} A^{n-1}$ with

$$
A=\left(\begin{array}{llll}
1 & 1 & 0 & 0 \\
1 & 2 & 0 & 0 \\
0 & 0 & 1 & 1 \\
0 & 0 & 1 & 2
\end{array}\right)
$$

Diagonalizing

$$
B=\left(\begin{array}{ll}
1 & 1 \\
1 & 2
\end{array}\right)
$$

we obtain $B=P D P^{-1}$ with $P^{t}=P^{-1}$ and

$$
D=\left(\begin{array}{cc}
\tau_{+}^{2} & 0 \\
0 & \tau_{-}^{2}
\end{array}\right), \quad P=\left(\begin{array}{cc}
\frac{1}{\sqrt{1+\tau_{+}^{2}}} & \frac{1}{\sqrt{1+\tau^{2}}} \\
\frac{\tau_{+}}{\sqrt{1+\tau_{+}^{2}}} & \frac{\tau_{-}}{\sqrt{1+\tau^{2}}}
\end{array}\right) .
$$


Since we are considering periodic boundary conditions, the rank of $\rho^{(N)}$ is exactly $x_{00}^{n}+x_{11}^{n}=$

$$
x^{1}\left(\begin{array}{cc}
P & 0 \\
0 & P
\end{array}\right)\left(\begin{array}{cc}
D^{n-1} & 0 \\
0 & D^{n-1}
\end{array}\right)\left(\begin{array}{cc}
P^{t} & 0 \\
0 & P^{t}
\end{array}\right)\left(\begin{array}{l}
1 \\
0 \\
0 \\
1
\end{array}\right) .
$$

Using the defining property $\tau_{ \pm}^{2}=\tau_{ \pm}+1$ it is an straightforward computation to verify that (D.6) is exactly

$$
\tau_{+}^{2 N}+\tau_{-}^{2 N}
$$

\section{Appendix D.2. General relations between NNPCH and correlation functions}

In Section 3 (see Theorem 3.10) we have shown that for a spin chain, ZCL of a tensor is equivalent to the existence of a NNPCH for the generated states. In this appendix we show that there is a deeper relation between those concepts which is independent of the dimension or the fact that states are generated by tensor networks.

Let us consider a spin system in any dimension and two disjoint regions, $A$ and $B$. We are interested in analyzing the correlations between operators acting on those regions for certain states. We will denote by $X$ the spins not included in $A$ or $B$, and by $H_{A, X, B}$, the corresponding Hilbert spaces. We will also denote by $H_{A X}=H_{A} \otimes H_{X}$, and by $H_{X B}=H_{X} \otimes H_{B}$. Let us consider $K_{A X B}$, a subspace of the whole Hilbert space and denote by $P_{A X B}$ the projector onto that subspace.

Definition D.1. Given a subspace $K_{A X B}$ we say that regions $A$ and $B$ are decorrelated if for all observables $O_{A, B}$ acting on $H_{A, B}$, respectively,

$$
P_{A X B} O_{A} P_{A X B}^{\perp} O_{B} P_{A X B}=0 .
$$

Note that we can equivalently write

$$
\left\langle\varphi\left|O_{A} P_{A X B}^{\perp} O_{B}\right| \varphi^{\prime}\right\rangle=\left\langle\varphi\left|O_{B} P_{A X B}^{\perp} O_{A}\right| \varphi^{\prime}\right\rangle=0
$$

for all $\varphi, \varphi^{\prime} \in K_{A X B}$. In particular, if the subspace has just one vector, $\varphi$, we will have

$$
\left\langle O_{A} O_{B}\right\rangle=\left\langle O_{A}\right\rangle\left\langle O_{B}\right\rangle
$$

where the expectation value is taken with respect to $\varphi$. This definition of correlations has been used, for example, in [41].

Our aim is to connect this concept of correlations with the fact that $K_{A X B}$ is the ground space of certain Hamiltonian, $H$, whenever $A$ and $B$ are not neighbors. We take $H$ local in the sense that it can be written as $H=Q_{A X}+$ $Q_{X B}$, where $Q_{A X}\left(Q_{X B}\right)$ acts on $H_{A X}\left(H_{X B}\right)$. We will consider frustration-free problems, so that we can take wlog $Q_{A X}$ and $Q_{X B}$ to be projectors. We will denote by $K_{A X}$ and $K_{X B}$ the kernels of $Q_{A X}$ and $Q_{X B}$, respectively. 
Definition D.2. Given a subspace $K_{A X B}$ we say that it corresponds to a parent commuting Hamiltonian if there exist two projectors, $Q_{A X}$ and $Q_{X B}$, acting on $H_{A X}$ and $H_{X B}$ respectively, such that

$$
\begin{aligned}
{\left[Q_{A X}, Q_{X B}\right] } & =0 \\
K_{A X B} & =\left(K_{A X} \otimes H_{B}\right) \cap\left(H_{A} \otimes K_{X B}\right)
\end{aligned}
$$

Note that the last property (D.10b) means that $K_{A X B}$ coincides with the ground subspace of $H=Q_{A X}+Q_{X B}$. In the following we will show that the two definitions given above are equivalent.

Proposition D.3. Given the subspace $K_{A X B}$, it corresponds to a parent commuting Hamiltonian if and only if regions $A$ and $B$ are decorrelated.

Proof. (if): Let us denote by $K_{A X}$ the support of $\operatorname{tr}_{B}\left(K_{A X B}\right)$ and by $K_{X B}$ the support of $\operatorname{tr}_{A}\left(K_{A X B}\right)$, and by $P_{A X}$ and $P_{X B}$ the corresponding projectors. Obviously, if $\varphi \in K_{A X B}$ we will have

$$
|\varphi\rangle=P_{A X}|\varphi\rangle=P_{X B}|\varphi\rangle
$$

so that

$$
P_{\alpha} P_{A X B}=P_{A X B} P_{\alpha}=P_{A X B} .
$$

where $\alpha=A X, X B$. Now, we choose orthonormal bases $\left\{a_{i}\right\} \in H_{A},\left\{b_{i}\right\} \in H_{B}$, $\left\{\alpha_{i}\right\} \in H_{A X}$ and $\left\{\beta_{i}\right\} \in H_{X B}$. We can always find some vectors $\varphi_{a, i}, \varphi_{b, i} \in$ $K_{A X B}, \tilde{a}_{i} \in H_{A}$ and $\tilde{b}_{i} \in H_{B}$ such that

$$
\begin{aligned}
\left|\alpha_{i}\right\rangle & =\left\langle\tilde{b}_{i} \mid \varphi_{b, i}\right\rangle, \\
\left|\beta_{i}\right\rangle & =\left\langle\tilde{a}_{i} \mid \varphi_{a, i}\right\rangle .
\end{aligned}
$$

We can thus write

$$
\begin{aligned}
P_{A X} \otimes \mathbb{1}_{B} & =\sum_{i, j}\left\langle\tilde{b}_{i} \mid \varphi_{b, i}\right\rangle\left\langle\varphi_{b, i} \mid \tilde{b}_{i}\right\rangle \otimes\left|b_{j}\right\rangle\left\langle b_{j}\right| \\
& =\sum_{i, j}\left|b_{j}\right\rangle_{B}\left\langle\tilde{b}_{i} \mid \varphi_{b, i}\right\rangle\left\langle\varphi_{b, i} \mid \tilde{b}_{i}\right\rangle_{B}\left\langle b_{j}\right|, \\
\mathbb{1}_{A} \otimes P_{X B} & =\sum_{i^{\prime}, j^{\prime}}\left|a_{j^{\prime}}\right\rangle\left\langle a_{j^{\prime}}\right| \otimes\left\langle\tilde{a}_{i^{\prime}} \mid \varphi_{a, i^{\prime}}\right\rangle\left\langle\varphi_{a, i^{\prime}} \mid \tilde{a}_{i^{\prime}}\right\rangle \\
& =\sum_{i^{\prime}, j^{\prime}}\left|a_{j^{\prime}}\right\rangle_{A}\left\langle\tilde{a}_{i^{\prime}} \mid \varphi_{a, i^{\prime}}\right\rangle\left\langle\varphi_{a, i^{\prime}} \mid \tilde{a}_{i^{\prime}}\right\rangle_{A}\left\langle a_{j^{\prime}}\right|
\end{aligned}
$$

where we have used the subindices $A, B$ in order to emphasize that the operators act on the corresponding regions. Using these expression we have

$$
\begin{aligned}
& P_{X B} P_{A X}=P_{X B} P_{A X B} P_{A X}=P_{A X B} \\
& P_{A X} P_{X B}=P_{A X} P_{A X B} P_{X B}=P_{A X B} .
\end{aligned}
$$


where we have used (D.12) as well as (D.8) with $\varphi=\varphi_{b, i}, \varphi^{\prime}=\varphi_{a, i^{\prime}}, O_{A}=$ $\left|a_{j^{\prime}}\right\rangle\left\langle\tilde{a}_{i^{\prime}}\right|$ and $O_{B}=\left|\tilde{b}_{i}\right\rangle\left\langle b_{j}\right|$. We thus arrive at $P_{A X} P_{X B}=P_{X B} P_{A X}$, which implies (D.10a) if we define $Q_{A X}$ and $Q_{X B}$ through

$$
\begin{aligned}
& P_{A X}=\mathbb{1}-Q_{A X}, \\
& P_{X B}=\mathbb{1}-Q_{X B} .
\end{aligned}
$$

Furthermore, from (D.11) it immediately follows that

$$
K_{A X B} \subseteq\left(K_{A X} \otimes H_{B}\right) \cap\left(H_{A} \otimes K_{X B}\right) .
$$

The converse relation can be proven as follows. Let us take any $\varphi \in K_{A X} \otimes H_{B}$ and $\varphi \in H_{A} \otimes K_{X B}$. Thus,

$$
|\varphi\rangle=P_{A X}|\varphi\rangle=P_{B X}|\varphi\rangle=P_{X B} P_{A X}|\varphi\rangle=P_{A X B}|\varphi\rangle,
$$

where we have used (D.15). Thus, $\varphi \in K_{A X B}$.

(only if): Let us define (D.16a,D.16b), and $P=P_{A X} P_{X B}$. Given that $K_{A X B} \subseteq\left(K_{A X} \otimes H_{B}\right) \cap\left(H_{A} \otimes K_{X B}\right)$ we have that if $\varphi \in K_{A X B}$, then (D.11) and thus (D.12). We have to show that

$$
P=P_{A X} P_{X B}=P_{A X B}
$$

In such case, for any $O_{A, B}$ acting on $H_{A, B}$, respectively, we have:

$$
\begin{aligned}
P_{A X B} O_{A} P_{A X B}^{\perp} O_{B} P_{A X B} & =P_{A X B} P_{X B} O_{A} P_{A X B}^{\perp} O_{B} P_{A X} P_{A X B} \\
& =P_{A X B} O_{A} P_{X B} P_{A X B}^{\perp} P_{A X} O_{B} P_{A X B}=0 \text { (D.20) }
\end{aligned}
$$

since $P_{X B} P_{A X B}^{\perp} P_{A X}=P_{X B}\left(\mathbb{1}-P_{A X B}\right) P_{A X}=P_{A X B}-P_{A X B}=0$, where we have used (D.19) and (D.12). In order to show (D.19), we recall that since $\left[P_{A X}, P_{X B}\right]=0, P$ must be a projector itself, i.e. $P=P^{\dagger}=P^{2}$. Furthermore, if $P_{A X B}|\varphi\rangle=|\varphi\rangle$, then we have (D.11) and thus $P|\varphi\rangle=|\varphi\rangle$. Conversely, if $P|\varphi\rangle=$ $|\varphi\rangle$ then $Q_{A X}|\varphi\rangle=Q_{X B}|\varphi\rangle=0$, so that $\varphi \in\left(K_{A X} \otimes H_{B}\right) \cap\left(H_{A} \otimes K_{X B}\right)$. But then, according to (D.10b) $\varphi \in K_{A X B}$ so that $P_{A X B}|\varphi\rangle=|\varphi\rangle$.

\section{References}

[1] F. Verstraete, J. I. Cirac, V. Murg, Adv. Phys. 57,143 (2008).

[2] J. I. Cirac and F. Verstraete, J. Phys. A: Math. Theor. 42, 504004 (2009).

[3] S. R. White, Phys. Rev. Lett. 69, 2863 (1992).

[4] U. Schollwoeck, Rev. Mod. Phys. 77, 259 (2005).

[5] D. Perez-Garcia, M.M. Wolf, M. Sanz, F. Verstraete, J.I. Cirac, Phys. Rev. Lett. 100, 167202 (2008).

[6] L. Tagliacozzo, A. Celi, M. Lewenstein, Phys. Rev. X 4, 041024 (2014). 
[7] J. Haegeman, K. Van Acoleyen, N. Schuch, J. I. Cirac, F. Verstraete, Phys. Rev. X 5, 011024 (2015).

[8] N. Schuch, J.I. Cirac, D. Perez-Garcia, Annals of Physics 325, 2153 (2010).

[9] M.B. Sahinoglu, D. Williamson, N. Bultinck, M. Marin, J. Haegeman, N. Schuch, and F. Verstraete, arXiv:1409.2150.

[10] X. Chen, Z.-C. Gu, Z.-X. Liu, X.-G. Wen, Phys. Rev. B 87, 155114 (2013).

[11] X. Chen, Z.-C. Gu, X.-G. Wen, Phys. Rev. B 83, 035107 (2011).

[12] N. Schuch, D. Perez-Garcia, J. I. Cirac, Phys. Rev. B 84, 165139 (2011)

[13] F. Pollmann, A. M. Turner, E. Berg, M. Oshikawa, Phys. Rev. B 81, 064439 (2010).

[14] F. Verstraete, J.I. Cirac, J.I. Latorre, E. Rico, M.M. Wolf, Phys. Rev. Lett. 94, 140601 (2005).

[15] G. Vidal, Phys. Rev. Lett. 99, 220405 (2007).

[16] Z.-C. Gu, M. Levin, X.-G. Wen, Phys. Rev. B 78, 205116 (2008).

[17] G. Evenbly, G. Vidal Phys. Rev. Lett. 115, 180405 (2015); ibid. 115, 200401 (2015).

[18] Z.-C. Gu, Z. Wang, X.-G. Wen, Phys. Rev. B 91, 125149 (2015).

[19] A. Kitaev, Ann. Phys. 303, 2 (2003),

[20] M.A. Levin, X.-G. Wen, Phys. Rev. B 71, 045110 (2005).

[21] F. Verstraete, J. I. Cirac, (2004), cond-mat/0407066; F. Verstraete, J. I. Cirac, Phys. Rev. B 73, 094423 (2006).

[22] F. Verstraete, M. M. Wolf, D. Perez-Garcia, J. I. Cirac, Phys. Rev. Lett. 96, 220601 (2006); D. Perez-Garcia, F. Verstraete, J. I. Cirac, M. M. Wolf, Quant. Inf. Comp. 8, 650 (2008).

[23] N. Bultinck, M. Marin, D. J. Williamson, M. B. Sahinoglu, J. Haegeman, F. Verstraete, arXiv:1511.08090.

[24] J. I. Cirac, D. Poilblanc, N. Schuch, Frank Verstraete, Phys. Rev. B 83, 245134 (2011).

[25] S. Ryu, J. E. Moore, A. W. W. Ludwig, Phys. Rev. B 85, 045104 (2012).

[26] N. Schuch, D. Poilblanc, J. I. Cirac, D. Perez-Garcia, Phys. Rev. Lett. 111, 090501 (2013).

[27] D. Perez-Garcia, F. Verstraete, M.M. Wolf, J.I. Cirac, Quantum Inf. Comput. 7, 401 (2007). 
[28] M. Fannes, B. Nachtergaele, R. F. Werner, Commun. Math. Phys. 114 (1992), 443-490.

[29] M. Sanz, D. Perez-Garcia, M.M. Wolf, J.I. Cirac, IEEE Trans. Inf. Theory 56 (2010) 4668-4673.

[30] F. Verstraete, J.J. Garcia-Ripoll, J.I. Cirac, Phys. Rev. Lett. 93, 207204 (2004).

[31] M. Zwolak and G. Vidal, Phys. Rev. Lett. 93, 207205 (2004).

[32] H. Li, F.D.M. Haldane, Phys. Rev. Lett. 101, 010504 (2008).

[33] G. De las Cuevas, T. S. Cubitt, J. I. Cirac, M. M. Wolf, D. Perez-Garcia, arXiv:1512.05709.

[34] M.M. Wolf, Quantum Channels and Operations: Guided Tour; available at http://www-m5.ma.tum.de/Allgemeines/MichaelWolf.

[35] S. Beigi, J. Phys. A: Math. Theor. 45 (2012) 025306.

[36] M.M. Wolf, F. Verstraete, M.B. Hastings, J.I. Cirac, Phys. Rev. Lett. 100, 070502 (2008).

[37] R.A. Horn, C. R. Johnson, Matrix Analysis, Cambridge University Press, (1985).

[38] M.M. Wolf, D. Perez-Garcia, arXiv:1005.4545.

[39] S. Bravyi, M. Vyalyi, Quantum Inf. and Comp., Vol. 5, No. 3 (2005) pp.187215.

[40] P. Hayden, R. Jozsa, D. Petz, A. Winter, Commun. Math. Phys., 246(2):359-374, 2004.

[41] M.B. Hastings and T. Koma, Commun. Math. Phys. 265, 781 (2006). 